Article

\title{
A New Algorithm for the Common Solutions of a Generalized Variational Inequality System and a Nonlinear Operator Equation in Banach Spaces
}

\author{
Yuanheng Wang * ${ }^{(\mathbb{O}}$, Cancan $\mathrm{Li}^{\dagger}$ and Lirong $\mathrm{Lu}^{\dagger}$ \\ College of Mathematics and Computer Science, with College of Xingzhi, Zhejiang Normal University, \\ 268 Yingbin Road, Jinhua 321004, Zhejiang, China; ccli521133@163.com (C.L.); yhwang@zjnu.edu.cn (L.L.) \\ * Correspondence: yhwang@zjnu.cn; Tel.: +86-579-8229-8258 \\ + These authors contributed equally to this work.
}

Received: 2 September 2020 ; Accepted: 27 October 2020 ; Published: 4 November 2020 updates

\begin{abstract}
We study a new algorithm for the common solutions of a generalized variational inequality system and the fixed points of an asymptotically non-expansive mapping in Banach spaces. Under some specific assumptions imposed on the control parameters, some strong convergence theorems for the sequence generated by our new viscosity iterative scheme to approximate their common solutions are proved. As an application of our main results, we solve the standard constrained convex optimization problem. The results here generalize and improve some other authors' recently corresponding results.
\end{abstract}

Keywords: asymptotically non-expansive mapping; general variational inequality system; strong convergence; Banach space

MSC: 47H10; 47H09; 47H05; 47J25

\section{Introduction}

In the paper, we assume that $X$ is a real Banach space and $X^{*}$ is the dual space of $X$. The normalized duality mapping $J: X \rightarrow 2^{X^{*}}$ is defined by

$$
J(v)=\left\{f \in X^{*}:\langle v, f\rangle=\|v\|\|f\|,\|v\|=\|f\|\right\}, \forall v \in X,
$$

where $\langle\cdot, \cdot\rangle$ denotes the duality pairing between $X$ and $X^{*}$. As we all know, the mapping $J$ is well defined and $J$ is identity mapping if and only if $X$ is a Hilbert space. In general, $J$ is multiple-valued and nonlinear, $j \in J$.

A Banach space $X$ is called strictly convex if $\frac{\|x+y\|}{2}<1$ for $\|x\|=\|y\|=1$ and $x \neq y$. The modulus of convexity of $X$ is defined by

$$
\delta_{X}(\epsilon)=\inf \left\{1-\left\|\frac{1}{2}(v+u)\right\|:\|v\|=\|u\| \leq 1,\|v-u\|=\epsilon\right\},
$$

for all $0<\epsilon \leq 2$. $X$ is said to be uniformly convex if $\delta_{X}(\epsilon)>0$ for all $0<\epsilon \leq 2$.

Let $\rho_{X}:[0,+\infty) \longrightarrow[0,+\infty)$ be the modulus of smoothness of $X$ defined by

$$
\rho_{X}(\zeta)=\sup \left\{\frac{1}{2}(\|v+u\|+\|v-u\|)-1:\|v\|=1 ;\|u\|=\zeta\right\} .
$$


A Banach space $X$ is called uniformly smooth if $\frac{\rho_{X}(\zeta)}{\zeta} \rightarrow 0$ as $\zeta \rightarrow 0$. Moreover, $X$ is uniformly smooth if the norm of $X$ is uniformly Fréchet differentiable.

Suppose $\varnothing \neq C \subset X, T: C \rightarrow C$ is an operator and the set of fixed points of $T$ is denoted by $\operatorname{Fix}(T)$, that is $\operatorname{Fix}(T)=\{v \in C: v=T v\}$. $T$ is said to be asymptotically non-expansive, if there exists a real sequence $\left\{k_{n}\right\} \subset[1, \infty)$ with $\lim _{n \rightarrow \infty} k_{n}=1$ such that

$$
\left\|T^{n} v-T^{n} u\right\| \leq k_{n}\|v-u\|, \forall v, u \in C
$$

If $k_{n} \equiv 1$, then we call that $T$ is non-expansive. $T$ is said to be uniformly asymptotically regular iff $\lim _{n \rightarrow \infty}\left\|T^{n+1} v-T^{n} v\right\|=0, \forall v \in C$. T is said to be a contraction when there exists a number $\eta \in(0,1)$ such that $\|T v-T u\| \leq \eta\|v-u\|, \forall v, u \in C$.

A mapping $\widehat{B}: C \rightarrow X$ is called inverse strongly accretive if there exists $e \in R^{+}$such that

$$
\langle\widehat{B} v-\widehat{B} u, j(v-u)\rangle \geq e\|\widehat{B} v-\widehat{B} u\|^{2}, \forall v, u \in C .
$$

A bounded linear operator $\widehat{B}$ on $X$ is called strongly positive, if there exists a fixed number $\bar{\delta}>0$ such that

$$
\langle\widehat{B} v, j(v)\rangle \geq \bar{\delta}\|v\|^{2},\left\|e_{1} I-e_{2} \widehat{B}\right\|=\sup _{\|v\| \leq 1}\left|\left\langle\left(e_{1} I-e_{2} \widehat{B}\right) v, j(v)\right\rangle\right|, e_{1} \in[0,1], e_{2} \in[-1,1], \forall v \in X
$$

Assume $E \neq \varnothing, E \subset C \subseteq X$ is a convex and closed set. An operator $Q: C \rightarrow E$ is called sunny when $Q$ has the following relation:

$$
Q(v+\alpha(v-Q(v)))=Q(v), \forall v \in C, \alpha \geq 0,
$$

for $v+\alpha(v-Q(v)) \in C$. A mapping $Q: C \rightarrow E$ is called retraction if $Q v=v, \forall v \in E$. Moreover, $Q$ is called sunny non-expansive retraction from $C$ onto $E$ iff $Q$ is a sunny, retraction, and non-expansive operator from $C$ onto $E$.

Let $C$ be a nonempty closed convex subset of $X$ and $A, B: C \rightarrow X$ be two nonlinear mappings, respectively. Recall the classical variational inequality problem is to find a point $p \in C$ such that

$$
\langle A p, j(v-p)\rangle \geq 0, \forall v \in C
$$

The set of the solutions of the variational inequality (1) is denoted by $V I(C, A)$. Lots of problems in physics, optimization, differential equation (inclusion), finance and minimax problem reduce to find an element of (1) and relevant numerical analysis methods can be considered to solve the problems, see [1-16].

In 2011, Katchang et al. [17] introduced the following system of general variational inequalities. They considered the problem of finding $(t, s) \in C \times C$ such that:

$$
\left\{\begin{array}{l}
\langle\lambda A s+t-s, j(v-t)\rangle \geq 0, \forall v \in C \\
\langle\mu B t+s-t, j(v-s)\rangle \geq 0, \forall v \in C
\end{array}\right.
$$

and proved a strong convergence theorem for the following sequence of problem (2):

$$
\left\{\begin{array}{l}
u_{n}=Q_{C}(I-\mu B) v_{n} \\
v_{n+1}=\alpha_{n} f\left(v_{n}\right)+\beta_{n} v_{n}+\gamma_{n}\left[\beta S v_{n}+(1-\beta) Q_{C}(I-\lambda A) u_{n}\right]
\end{array}\right.
$$

where $\left\{\alpha_{n}\right\},\left\{\beta_{n}\right\},\left\{\gamma_{n}\right\} \subset(0,1), \lambda$ and $\mu$ are two positive real numbers, $0<\beta<1, Q_{C}$ is the sunny non-expansive retraction from $X$ to $C, f$ is a contractive mapping on $C$ and $S: C \rightarrow C$ is a non-expansive mapping. 
In 2020, Wang et al. [18] investigated a new general variational inequality system:

$$
\left\{\begin{array}{l}
\langle(I-\lambda A)(b t+(1-b) s)-t, j(v-t)\rangle \leq 0, \forall v \in C, \\
\langle(I-\mu B) t-s, j(v-s)\rangle \leq 0, \forall v \in C,
\end{array}\right.
$$

and proposed the following iterative method:

$$
\left\{\begin{array}{l}
w_{n}=Q_{C}(I-\mu B) v_{n}, \\
l_{n}=Q_{C}(I-\lambda A)\left(b v_{n}+(1-b) l_{n}\right), \\
u_{n}=\delta_{n} v_{n}+\left(1-\delta_{n}\right) l_{n}, \\
v_{n+1}=\alpha_{n} f\left(v_{n}\right)+\beta_{n} v_{n}+\gamma_{n} T^{n} u_{n},
\end{array}\right.
$$

where $\left\{\alpha_{n}\right\},\left\{\beta_{n}\right\},\left\{\gamma_{n}\right\},\left\{\delta_{n}\right\} \subset(0,1), \lambda$ and $\mu$ are two positive real numbers, $0 \leq b \leq 1, Q_{C}$ is the sunny non-expansive retraction from $X$ to $C, f$ is a contractive mapping on $C$ and $T: C \rightarrow C$ is an asymptotically non-expansive mapping. Then they proved that $v_{n}$ converges to the common element of the fixed points of an asymptotically non-expansive mapping and the solutions of the general variational inequality system (3).

Inspired by the above mentioned results, we study the following new generalized variational inequality system in uniformly convex and uniformly smooth Banach spaces:

$$
\left\{\begin{array}{l}
\left\langle t-\left(I-\lambda_{1} B_{1}\right)(b t+(1-b) s), j(v-t)\right\rangle \geq 0, \forall v \in C \\
\left\langle s-\left(I-\lambda_{2} B_{2}\right)(b t+(1-b) w), j(v-s)\right\rangle \geq 0, \forall v \in C \\
\left\langle w-\left(I-\lambda_{3} B_{3}\right) t, j(v-w)\right\rangle \geq 0, \forall v \in C .
\end{array}\right.
$$

This is the main problem which we seek to solve in this work and construct the suitable abstract iterative method. The new generalized variational inequality system problem (4) contains (1)-(3) as special cases. We construct a new iterative algorithm to approximate the common solutions of the generalized system of variational inequality (4) and an asymptotically non-expansive mapping. Some strong convergence theorems that depend on some suitable conditions are proved. Eventually, we show an application for solving the standard constrained problem of convex optimization to illustrate the efficiency of our main theorem. Some other outcomes proposed by other authors are also improved, see $[9,10,17-22]$.

\section{Preliminaries}

This section contains the useful lemmas that are indispensable for proving our convergence theorem in the following section. Generally speaking, we suppose that space $X$ is uniformly smooth and uniformly convex in this section.

Lemma 1 ([18]). Suppose that $X \supset C \neq \varnothing$ and $C$ is a closed convex set. Let $J$ be the normalized duality mapping $J: X \rightarrow 2^{X^{*}}$ and $Q: X \rightarrow C$ be a retraction. Then the followings are equivalent:

(I) $Q$ is sunny and non-expansive;

(II) $\langle v-Q v, J(u-Q v)\rangle \leq 0, \forall v \in X, u \in C$;

(III) $\|Q v-Q u\|^{2} \leq\langle v-u, J(Q v-Q u)\rangle, \forall v, u \in X$.

Lemma 2 ([23]). Assume $X$ is uniformly convex and $V_{R}(0)$ is a closed ball of $X$. Then there exists a continuous strictly increasing convex function $m_{1}:[0, \infty) \rightarrow[0, \infty)$ with $m_{1}(0)=0$ such that

$$
\left\|a_{1} v_{1}+a_{2} v_{2}+\cdots+a_{n} v_{n}\right\|^{2}=\sum_{i=1}^{n} a_{i}\left\|v_{i}\right\|^{2}-a_{x} a_{y} m_{1}\left(\left\|v_{x}-v_{y}\right\|\right),
$$


for any $x, y \in\{1,2, \ldots, n\}$ and for $v_{i} \in V_{R}(0):=\{v \in X:\|v\| \leq R\}, i=1,2, \ldots, n$ with $\sum_{i=1}^{n} a_{i}=1$.

Lemma 3 ([24]). Suppose $\left\{v_{n}\right\}$ and $\left\{z_{n}\right\}$ are two bounded sequences of $X$. Let $\left\{\mu_{n}\right\} \subset[0,1]$ be a number sequence with $0<\liminf _{n \rightarrow \infty} \mu_{n} \leq \limsup _{n \rightarrow \infty} \mu_{n}<1$. Assume that $v_{n+1}=\left(1-\mu_{n}\right) v_{n}+\mu_{n} z_{n}, n \geq 0$ and $\limsup _{n \rightarrow \infty}\left(\left\|z_{n+1}-z_{n}\right\|-\left\|v_{n+1}-v_{n}\right\|\right) \leq 0$. Then $\lim _{n \rightarrow \infty}\left\|z_{n}-v_{n}\right\|=0$.

Lemma 4 ([25]). For $\forall v, u \in X, j(v+u) \in J(v+u)$, there holds the relation:

$$
\|v+u\|^{2} \leq\|v\|^{2}+2\langle u, j(v+u)\rangle .
$$

Lemma 5 ([26]). Let $\left\{c_{n}\right\}$ be a nonnegative number sequence satisfying:

$$
c_{n+1} \leq\left(1-\sigma_{n}\right) c_{n}+\sigma_{n} \theta_{n}, \forall n \geq 0,
$$

where

$$
\left\{\sigma_{n}\right\} \subset[0,1], \sum_{n=0}^{\infty} \sigma_{n}=+\infty ; \limsup _{n \rightarrow \infty} \theta_{n} \leq 0 \text { or } \sum_{n=1}^{\infty}\left|\sigma_{n} \theta_{n}\right|<+\infty .
$$

Then $\lim _{n \rightarrow \infty} c_{n}=0$.

Lemma 6 ([27]). Suppose that $\bar{V}$ is a strongly positive linear bounded operator on $X$ with coefficient $\bar{\delta}>0$ and $0<\rho \leq\|\bar{V}\|^{-1}$. Then, $\|I-\rho \bar{V}\| \leq 1-\rho \bar{\delta}$.

Lemma 7 ([18]). Assume $C$ is a nonempty convex and closed subset of $X$. Assume mapping $\widehat{B}: C \rightarrow X$ is $a \widehat{b}$-inverse strongly accretive. Then,

$$
\|(I-\lambda \widehat{B}) v-(I-\lambda \widehat{B}) u\|^{2} \leq\|v-u\|^{2}-\lambda(2 \widehat{b}-\lambda)\|\widehat{B} v-\widehat{B} u\|^{2}, \lambda>0 .
$$

If $0<\lambda<2 \widehat{b}$, then $I-\lambda \widehat{B}$ is non-expansive.

Lemma 8 ([28]). Let $C \neq \varnothing$ be a closed convex subset of $X$. Suppose $Q_{C}: X \rightarrow C$ is a sunny non-expansive mapping and $\widehat{B}$ is an accretive operator from $C$ into $X$. Then,

$$
V I(C, \widehat{B})=\operatorname{Fix}\left(Q_{C}(I-\lambda \widehat{B})\right), \lambda>0,
$$

where $\operatorname{VI}(C, \widehat{B})=\{t \in C:\langle\widehat{B} t, j(v-t)\rangle \geq 0, \forall v \in C\}$.

Lemma 9 ([18]). There exists a convex continuous strictly increasing function $m_{2}:[0,2 r] \rightarrow R, m_{2}(0)=0$ such that

$$
m_{2}(\|v-u\|) \leq\|v\|^{2}-2\langle v, j(u)\rangle+\|u\|^{2}, \forall r>0, v, u \in B_{r} .
$$

Lemma 10 ([9]). Assume $\varnothing \neq C \subset X$ is a closed convex set. Suppose $T: C \rightarrow C$ is an asymptotically non-expansive mapping and Fix $(T) \neq \varnothing$. If $X$ has a weakly sequentially continuous duality mapping $J$, then the mapping $I-T$ is demi-closed at zero, where $I$ is the identity mapping, i.e., if $v_{n} \rightarrow v$ and $\left\|v_{n}-T v_{n}\right\| \rightarrow 0$, then $v=T v$.

Lemma 11. Assume $X \supset C \neq \varnothing$ is a closed convex set. Let $B_{1}, B_{2}, B_{3}: C \rightarrow X$ be three mappings, respectively. When $\lambda_{1}, \lambda_{2}, \lambda_{3}>0, b \in[0,1)$, then the following statements are the same:

(i) $(t, s, w) \in C \times C \times C$ is a solution of the problem (4);

(ii) $t$ is a fixed point of the mapping $L$, i.e., $t \in$ Fix $(L)$, where $L: C \rightarrow C$ is defined by

$$
L(v)=Q_{C}\left(I-\lambda_{1} B_{1}\right)\left(b v+(1-b) Q_{C}\left(I-\lambda_{2} B_{2}\right)\left(b v+(1-b) Q_{C}\left(I-\lambda_{3} B_{3}\right) v\right)\right), \forall v \in C,
$$


and $s=Q_{C}\left(I-\lambda_{2} B_{2}\right)(b t+(1-b) w), w=Q_{C}\left(I-\lambda_{3} B_{3}\right) t$.

Proof. $(i) \Rightarrow(i i)$. Assume that $(t, s, w) \in C \times C \times C$ is a solution of (4). For all $v \in C$, we have

$$
\begin{aligned}
& \left\langle t-\left(I-\lambda_{1} B_{1}\right)(b t+(1-b) s), j(v-t)\right\rangle \geq 0 \\
& \left\langle s-\left(I-\lambda_{2} B_{2}\right)(b t+(1-b) w), j(v-s)\right\rangle \geq 0 \\
& \left\langle w-\left(I-\lambda_{3} B_{3}\right) t, j(v-w)\right\rangle \geq 0 .
\end{aligned}
$$

From property of $Q_{C}$, we get

$$
\begin{aligned}
& t=Q_{C}\left(I-\lambda_{1} B_{1}\right)(b t+(1-b) s) \\
& s=Q_{C}\left(I-\lambda_{2} B_{2}\right)(b t+(1-b) w), \\
& w=Q_{C}\left(I-\lambda_{3} B_{3}\right) t
\end{aligned}
$$

It implies that

$$
t=Q_{C}\left(I-\lambda_{1} B_{1}\right)\left(b t+(1-b) Q_{C}\left(I-\lambda_{2} B_{2}\right)\left(b t+(1-b) Q_{C}\left(I-\lambda_{3} B_{3}\right) t\right)\right)=L(t) .
$$

Then $t \in$ Fix $(L)$, where $s=Q_{C}\left(I-\lambda_{2} B_{2}\right)(b t+(1-b) w)$ and $w=Q_{C}\left(I-\lambda_{3} B_{3}\right) t$.

(ii) $\Rightarrow($ i $)$. Let $t \in$ Fix $(L), s=Q_{C}\left(I-\lambda_{2} B_{2}\right)(b t+(1-b) w)$ and $w=Q_{C}\left(I-\lambda_{3} B_{3}\right) t$. Since $t \in \operatorname{Fix}(L)$, we have

$$
\begin{aligned}
t & =Q_{C}\left(I-\lambda_{1} B_{1}\right)\left(b t+(1-b) Q_{C}\left(I-\lambda_{2} B_{2}\right)\left(b t+(1-b) Q_{C}\left(I-\lambda_{3} B_{3}\right) t\right)\right) \\
& =Q_{C}\left(I-\lambda_{1} B_{1}\right)(b t+(1-b) s) .
\end{aligned}
$$

In combination with $s=Q_{C}\left(I-\lambda_{2} B_{2}\right)(b t+(1-b) w), w=Q_{C}\left(I-\lambda_{3} B_{3}\right) t$ and the property of $Q_{C}$, we get

$$
\begin{aligned}
& \left\langle t-\left(I-\lambda_{1} B_{1}\right)(b t+(1-b) s), j(v-t)\right\rangle \geq 0, \forall v \in C, \\
& \left\langle s-\left(I-\lambda_{2} B_{2}\right)(b t+(1-b) w), j(v-s)\right\rangle \geq 0, \forall v \in C, \\
& \left\langle w-\left(I-\lambda_{3} B_{3}\right) t, j(v-w)\right\rangle \geq 0, \forall v \in C .
\end{aligned}
$$

Then $(t, s, w) \in C \times C \times C$ is a solution of the problem (4).

\section{Results}

Theorem 1. Suppose $X$ is a uniformly smooth and uniformly convex Banach space and $C \neq \varnothing$ is a closed convex subset of $X$. Assume $Q_{C}: X \rightarrow C$ is a sunny non-expansive retraction. Let $\widehat{B}, B_{1}, B_{2}, B_{3}: C \rightarrow$ $X$ be $\widehat{b}, b_{1}, b_{2}, b_{3}$-inverse strongly accretive, respectively. L is the mapping defined by (5) in Lemma 11 and $b \in[0,1)$. Assume $\bar{V}: X \rightarrow X$ is a bounded linear strongly positive operator on $X$ with coefficient $\bar{\delta}>0$ and $0<\bar{\delta}<\|\bar{V}\|^{-1}$. Let $g$ be an $\eta \in(0,1)$-contraction mapping on $C$ and $T: C \rightarrow C$ be an asymptotically non-expansive mapping with $\Psi=\operatorname{Fix}(T) \cap \operatorname{Fix}(L) \cap V I(C, \widehat{B}) \neq \varnothing$. Assume $\left\{v_{n}\right\}$ is defined as follows:

$$
\left\{\begin{array}{l}
v_{1} \in C \\
u_{n}=\beta_{n} \delta g\left(v_{n}\right)+\left(I-\beta_{n} \bar{V}\right) L v_{n} \\
v_{n+1}=\mu_{n} v_{n}+v_{n} T^{n}\left(\xi_{n} v_{n}+\left(1-\xi_{n}\right) v_{n+1}\right)+\omega_{n} Q_{C}(I-\lambda \widehat{B}) u_{n}, n \geq 0,
\end{array}\right.
$$

where $\lambda \in(0,2 \widehat{b}),\left\{\beta_{n}\right\} \subset(0,1), \mu_{n}+v_{n}+\omega_{n}=1$, and the following coefficient conditions are satisfying: 
(i) $\lim _{n \rightarrow \infty} \beta_{n}=0, \sum_{n=0}^{\infty} \beta_{n}=\infty, \sum_{n=1}^{\infty}\left|\beta_{n+1}-\beta_{n}\right|<\infty$;

(ii) $0<h_{1} \leq \mu_{n}, v_{n}, \omega_{n} \leq h_{2}<1, \sum_{n=1}^{\infty}\left|\mu_{n+1}-\mu_{n}\right|<\infty, \sum_{n=1}^{\infty}\left|v_{n+1}-v_{n}\right|<\infty, \sum_{n=1}^{\infty}\left|\omega_{n+1}-\omega_{n}\right|<\infty$;

(iii) $0 \leq \xi_{n} \leq \xi_{n+1} \leq 1,0<\delta<\frac{h_{1} \bar{\delta}}{2 \eta}, k_{n}^{2}-1=\varepsilon \beta_{n}, 0<\varepsilon<h_{1} \bar{\delta}-2 h_{1} \delta \eta$;

(iv) $v_{n} k_{n}^{2}\left(1-\xi_{n}\right)<1-\tau, h_{2}<\tau<1$.

Then the sequence $\left\{v_{n}\right\}$ converges strongly to $t=Q_{\Psi}(I-\bar{V}+\delta g) t \in \Psi$ and $(t, s, w)$ is a solution of (4), where $s=Q_{C}\left(I-\lambda_{2} B_{2}\right)(b t+(1-b) w)$ and $w=Q_{C}\left(I-\lambda_{3} B_{3}\right) t, \lambda_{1}, \lambda_{2}, \lambda_{3} \in(0,2 \bar{b})$ with $\bar{b}=\min \left\{b_{1}, b_{2}, b_{3}\right\}$.

Proof. Our proof process can be divided into 6 steps.

Step 1: Let $\widehat{B}, B_{1}, B_{2}, B_{3}$ be $\widehat{b}, b_{1}, b_{2}, b_{3}$-inverse strongly accretive mapping, respectively, and $\lambda \in(0,2 \widehat{b}), \lambda_{1}, \lambda_{2}, \lambda_{3} \in(0,2 \bar{b})$ with $\bar{b}=\min \left\{b_{1}, b_{2}, b_{3}\right\}$. From Lemma 7 , we know that $(I-\lambda \widehat{B}),\left(I-\lambda_{1} B_{1}\right),\left(I-\lambda_{2} B_{2}\right)$ and $\left(I-\lambda_{3} B_{3}\right)$ are non-expansive mappings. Moveover, we attain that $Q_{C}(I-\lambda \widehat{B}), Q_{C}\left(I-\lambda_{1} B_{1}\right), Q_{C}\left(I-\lambda_{2} B_{2}\right)$ and $Q_{C}\left(I-\lambda_{3} B_{3}\right)$ are non-expansive mapping.

So, we have

$$
\begin{aligned}
\|L v-L u\|= & \| Q_{C}\left(I-\lambda_{1} B_{1}\right)\left(b v+(1-b) Q_{C}\left(I-\lambda_{2} B_{2}\right)\left(b v+(1-b) Q_{C}\left(I-\lambda_{3} B_{3}\right) v\right)\right) \\
& -Q_{C}\left(I-\lambda_{1} B_{1}\right)\left(b u+(1-b) Q_{C}\left(I-\lambda_{2} B_{2}\right)\left(b u+(1-b) Q_{C}\left(I-\lambda_{3} B_{3}\right) u\right)\right) \| \\
\leq & \|\left(b v+(1-b) Q_{C}\left(I-\lambda_{2} B_{2}\right)\left(b v+(1-b) Q_{C}\left(I-\lambda_{3} B_{3}\right) v\right)\right) \\
& -\left(b u+(1-b) Q_{C}\left(I-\lambda_{2} B_{2}\right)\left(b u+(1-b) Q_{C}\left(I-\lambda_{3} B_{3}\right) u\right)\right) \| \\
\leq & b\|v-u\|+(1-b) \| Q_{C}\left(I-\lambda_{2} B_{2}\right)\left(b v+(1-b) Q_{C}\left(I-\lambda_{3} B_{3}\right) v\right) \\
& -Q_{C}\left(I-\lambda_{2} B_{2}\right)\left(b u+(1-b) Q_{C}\left(I-\lambda_{3} B_{3}\right) u\right) \| \\
\leq & b\|v-u\|+(1-b)\left\|\left(b v+(1-b) Q_{C}\left(I-\lambda_{3} B_{3}\right) v\right)-\left(b u+(1-b) Q_{C}\left(I-\lambda_{3} B_{3}\right) u\right)\right\| \\
\leq & b\|v-u\|+(1-b)\left(b\|v-u\|+(1-b)\left\|Q_{C}\left(I-\lambda_{3} B_{3}\right) v-Q_{C}\left(I-\lambda_{3} B_{3}\right) u\right\|\right) \\
\leq & b\|v-u\|+(1-b)(b\|v-u\|+(1-b)\|v-u\|) \\
= & \|v-u\| .
\end{aligned}
$$

Then, $L$ is non-expansive.

Step 2: For any given $q \in \Psi$, from (6), we have

$$
\begin{aligned}
\left\|T^{n}\left(\xi_{n} v_{n}+\left(1-\xi_{n}\right) v_{n+1}\right)-q\right\| & \leq k_{n}\left\|\xi_{n} v_{n}+\left(1-\xi_{n}\right) v_{n+1}-q\right\| \\
& \leq k_{n} \xi_{n}\left\|v_{n}-q\right\|+k_{n}\left(1-\xi_{n}\right)\left\|v_{n+1}-q\right\|,
\end{aligned}
$$

and

$$
\begin{aligned}
\left\|u_{n}-q\right\| & =\left\|\beta_{n} \delta g\left(v_{n}\right)+\left(I-\beta_{n} \bar{V}\right) L v_{n}-q\right\| \\
& \leq \beta_{n}\left\|\delta g\left(v_{n}\right)-\bar{V} q\right\|+\left\|\left(I-\beta_{n} \bar{V}\right)\left(L v_{n}-q\right)\right\| \\
& \leq \beta_{n}\left\|\delta g\left(v_{n}\right)-\delta g(q)\right\|+\beta_{n}\|\delta g(q)-\bar{V} q\|+\left(1-\beta_{n} \bar{\delta}\right)\left\|v_{n}-q\right\| \\
& \leq \beta_{n} \delta \eta\left\|v_{n}-q\right\|+\beta_{n}\|\delta g(q)-\bar{V} q\|+\left(1-\beta_{n} \bar{\delta}\right)\left\|v_{n}-q\right\| \\
& \leq\left[1-\beta_{n}(\bar{\delta}-\delta \eta)\right]\left\|v_{n}-q\right\|+\beta_{n}\|\delta g(q)-\bar{V} q\| .
\end{aligned}
$$


Then we compute

$$
\begin{aligned}
\left\|v_{n+1}-q\right\|= & \left\|\mu_{n} v_{n}+v_{n} T^{n}\left(\xi_{n} v_{n}+\left(1-\xi_{n}\right) v_{n+1}\right)+\omega_{n} Q_{C}(I-\lambda \widehat{B}) u_{n}-q\right\| \\
\leq & \left\|\mu_{n}\left(v_{n}-q\right)+v_{n}\left(T^{n}\left(\xi_{n} v_{n}+\left(1-\xi_{n}\right) v_{n+1}\right)-q\right)+\omega_{n}\left(Q_{C}(I-\lambda \widehat{B}) u_{n}-q\right)\right\| \\
\leq & \mu_{n}\left\|v_{n}-q\right\|+v_{n}\left\|T^{n}\left(\xi_{n} v_{n}+\left(1-\xi_{n}\right) v_{n+1}\right)-q\right\|+\omega_{n}\left\|u_{n}-q\right\| \\
\leq & \mu_{n}\left\|v_{n}-q\right\|+v_{n} k_{n} \xi_{n}\left\|v_{n}-q\right\|+v_{n} k_{n}\left(1-\xi_{n}\right)\left\|v_{n+1}-q\right\| \\
& +\omega_{n}\left[1-\beta_{n}(\bar{\delta}-\delta \eta)\right]\left\|v_{n}-q\right\|+\omega_{n} \beta_{n}\|\delta g(q)-\bar{V} q\| \\
\leq & \left(\mu_{n}+v_{n} k_{n} \xi_{n}+\omega_{n}\left[1-\beta_{n}(\bar{\delta}-\delta \eta)\right]\right)\left\|v_{n}-q\right\|+v_{n} k_{n}\left(1-\xi_{n}\right)\left\|v_{n+1}-q\right\| \\
& +\omega_{n} \beta_{n}\|\delta g(q)-\bar{V} q\| .
\end{aligned}
$$

It follows that

$\left(1-v_{n} k_{n}\left(1-\xi_{n}\right)\right)\left\|v_{n+1}-q\right\| \leq\left(\mu_{n}+v_{n} k_{n} \xi_{n}+\omega_{n}\left[1-\beta_{n}(\bar{\delta}-\delta \eta)\right]\right)\left\|v_{n}-q\right\|+\omega_{n} \beta_{n}\|\delta g(q)-\bar{V} q\|$.

From conditions (ii), (iii) and (iv) in Theorem 1, we get

$$
\begin{aligned}
\left\|v_{n+1}-q\right\| & \leq \frac{\mu_{n}+v_{n} k_{n} \xi_{n}+\omega_{n}\left[1-\beta_{n}(\bar{\delta}-\delta \eta)\right]}{1-v_{n} k_{n}\left(1-\xi_{n}\right)}\left\|v_{n}-q\right\|+\frac{\omega_{n} \beta_{n}}{1-v_{n} k_{n}\left(1-\xi_{n}\right)}\|\delta g(q)-\bar{V} q\| \\
& \leq\left(1-\frac{\omega_{n} \beta_{n}(\bar{\delta}-\delta \eta)-v_{n}\left(k_{n}-1\right)}{1-v_{n} k_{n}\left(1-\xi_{n}\right)}\right)\left\|v_{n}-q\right\|+\frac{\omega_{n} \beta_{n}}{1-v_{n} k_{n}\left(1-\xi_{n}\right)}\|\delta g(q)-\bar{V} q\| \\
& \leq\left(1-\frac{h_{1} \beta_{n}(\bar{\delta}-\delta \eta)-\varepsilon \beta_{n}}{1-v_{n} k_{n}\left(1-\xi_{n}\right)}\right)\left\|v_{n}-q\right\|+\frac{\beta_{n}}{1-v_{n} k_{n}\left(1-\xi_{n}\right)}\|\delta g(q)-\bar{V} q\| \\
& \leq\left(1-\frac{\beta_{n}\left(h_{1} \bar{\delta}-h_{1} \delta \eta-\varepsilon\right)}{1-v_{n} k_{n}\left(1-\xi_{n}\right)}\right)\left\|v_{n}-q\right\|+\frac{\beta_{n}\left(h_{1} \bar{\delta}-h_{1} \delta \eta-\varepsilon\right)}{1-v_{n} k_{n}\left(1-\xi_{n}\right)} \frac{\|\delta g(q)-\bar{V} q\|}{h_{1} \bar{\delta}-h_{1} \delta \eta-\varepsilon} \\
& \leq \max \left\{\left\|v_{n}-q\right\|, \frac{\|\delta g(q)-\bar{V} q\|}{h_{1} \bar{\delta}-h_{1} \delta \eta-\varepsilon}\right\} .
\end{aligned}
$$

By induction, we readily obtain

$$
\left\|v_{n+1}-q\right\| \leq \max \left\{\left\|v_{1}-q\right\|, \frac{\|\delta g(q)-\bar{V} q\|}{h_{1} \bar{\delta}-h_{1} \delta \eta-\varepsilon}\right\} .
$$

This implies that $\left\{v_{n}\right\}$ is bounded, and so are $\left\{u_{n}\right\},\left\{g\left(v_{n}\right)\right\},\left\{L v_{n}\right\},\left\{T^{n}\left(\xi_{n} v_{n}+\left(1-\xi_{n}\right) v_{n+1}\right)\right\}$. Step 3: By (6) and Lemma 6, we gain that

$$
\begin{aligned}
\left\|u_{n+1}-u_{n}\right\|= & \left\|\beta_{n+1} \delta g\left(v_{n+1}\right)+\left(I-\beta_{n+1} \bar{V}\right) L v_{n+1}-\left(\beta_{n} \delta g\left(v_{n}\right)+\left(I-\beta_{n} \bar{V}\right) L v_{n}\right)\right\| \\
= & \| \beta_{n+1} \delta g\left(v_{n+1}\right)-\beta_{n+1} \delta g\left(v_{n}\right)+\beta_{n+1} \delta g\left(v_{n}\right)-\beta_{n} \delta g\left(v_{n}\right) \\
& +\left(I-\beta_{n+1} \bar{V}\right) L v_{n+1}-\left(I-\beta_{n+1} \bar{V}\right) L v_{n}+\left(I-\beta_{n+1} \bar{V}\right) L v_{n}-\left(I-\beta_{n} \bar{V}\right) L v_{n} \| \\
= & \| \beta_{n+1} \delta\left[g\left(v_{n+1}\right)-g\left(v_{n}\right)\right]+\left(\beta_{n+1}-\beta_{n}\right) \delta g\left(v_{n}\right)+\left(I-\beta_{n+1} \bar{V}\right)\left(L v_{n+1}-L v_{n}\right) \\
& -\left(\beta_{n+1}-\beta_{n}\right) \bar{V} L v_{n} \| \\
\leq & \beta_{n+1} \delta\left\|g\left(v_{n+1}\right)-g\left(v_{n}\right)\right\|+\left|\beta_{n+1}-\beta_{n}\right|\left\|\delta g\left(v_{n}\right)-\bar{V} L v_{n}\right\|+\left\|I-\beta_{n+1} \bar{V}\right\| \| L v_{n+1} \\
& -L v_{n} \| \\
\leq & \beta_{n+1} \delta \eta\left\|v_{n+1}-v_{n}\right\|+\left|\beta_{n+1}-\beta_{n}\right|\left\|\delta g\left(v_{n}\right)-\bar{V} L v_{n}\right\|+\left(1-\beta_{n+1} \bar{\delta}\right)\left\|v_{n+1}-v_{n}\right\| \\
= & \left(1-\beta_{n+1}(\bar{\delta}-\delta \eta)\right)\left\|v_{n+1}-v_{n}\right\|+\left|\beta_{n+1}-\beta_{n}\right|\left\|\delta g\left(v_{n}\right)-\bar{V} L v_{n}\right\| \\
\leq & \left\|v_{n+1}-v_{n}\right\|+\left|\beta_{n+1}-\beta_{n}\right|\left\|\delta g\left(v_{n}\right)-\bar{V} L v_{n}\right\| .
\end{aligned}
$$


By (6) and (7), we can acquire

$$
\begin{aligned}
& \left\|v_{n+2}-v_{n+1}\right\|=\| \mu_{n+1} v_{n+1}+v_{n+1} T^{n+1}\left(\xi_{n+1} v_{n+1}+\left(1-\xi_{n+1}\right) v_{n+2}\right)+\omega_{n+1} Q_{C}(I-\lambda \widehat{B}) u_{n+1} \\
& -\left[\mu_{n} v_{n}+v_{n} T^{n}\left(\xi_{n} v_{n}+\left(1-\xi_{n}\right) v_{n+1}\right)+\omega_{n} Q_{C}(I-\lambda \widehat{B}) u_{n}\right] \| \\
& =\| \mu_{n+1}\left(v_{n+1}-v_{n}\right)+\left(\mu_{n+1}-\mu_{n}\right) v_{n}+v_{n+1}\left(T^{n+1}\left(\xi_{n+1} v_{n+1}+\left(1-\xi_{n+1}\right) v_{n+2}\right)\right. \\
& \left.-T^{n+1}\left(\xi_{n} v_{n}+\left(1-\xi_{n}\right) v_{n+1}\right)\right)+\left(v_{n+1}-v_{n}\right) T^{n+1}\left(\xi_{n} v_{n}+\left(1-\xi_{n}\right) v_{n+1}\right) \\
& +v_{n}\left[T^{n+1}\left(\xi_{n} v_{n}+\left(1-\xi_{n}\right) v_{n+1}\right)-T^{n}\left(\xi_{n} v_{n}+\left(1-\xi_{n}\right) v_{n+1}\right)\right] \\
& +\omega_{n+1}\left[Q_{C}(I-\lambda \widehat{B}) u_{n+1}-Q_{C}(I-\lambda \widehat{B}) u_{n}\right]+\left(\omega_{n+1}-\omega_{n}\right) Q_{C}(I-\lambda \widehat{B}) u_{n} \| \\
& \leq \mu_{n+1}\left\|v_{n+1}-v_{n}\right\|+\left|\mu_{n+1}-\mu_{n}\right|\left\|v_{n}\right\|+v_{n+1} k_{n+1} \|\left(\xi_{n+1} v_{n+1}+\left(1-\xi_{n+1}\right) v_{n+2}\right) \\
& \left.-\left(\xi_{n} v_{n}+\left(1-\xi_{n}\right) v_{n+1}\right)\right)\left\|+\left|v_{n+1}-v_{n}\right|\right\| T^{n+1} v\left\|+v_{n} \sup _{v \in C}\right\| T^{n+1} v-T^{n} v \| \\
& +\omega_{n+1}\left\|Q_{C}(I-\lambda \widehat{B}) u_{n+1}-Q_{C}(I-\lambda \widehat{B}) u_{n}\right\|+\left|\omega_{n+1}-\omega_{n}\right|\left\|Q_{C}(I-\lambda \widehat{B}) u_{n}\right\| \\
& \leq \mu_{n+1}\left\|v_{n+1}-v_{n}\right\|+\left|\mu_{n+1}-\mu_{n}\right|\left\|v_{n}\right\|+v_{n+1} k_{n+1} \xi_{n}\left\|v_{n+1}-v_{n}\right\| \\
& +v_{n+1} k_{n+1}\left(1-\xi_{n+1}\right)\left\|v_{n+2}-v_{n+1}||+\left|v_{n+1}-v_{n}\right|\right\| T^{n+1} v \| \\
& +v_{n} \sup _{v \in C}\left\|T^{n+1} v-T^{n} v\right\|+\omega_{n+1}\left\|u_{n+1}-u_{n}\right\|+\left|\omega_{n+1}-\omega_{n}\right|\left\|Q_{C}(I-\lambda \widehat{B}) u_{n}\right\| \\
& \leq \mu_{n+1}\left\|v_{n+1}-v_{n}\right\|+\left|\mu_{n+1}-\mu_{n}\right|\left\|v_{n}\right\|+v_{n+1} k_{n+1} \xi_{n}\left\|v_{n+1}-v_{n}\right\| \\
& +v_{n+1} k_{n+1}\left(1-\xi_{n+1}\right)\left\|v_{n+2}-v_{n+1}\right\|+\left|v_{n+1}-v_{n}\right|\left\|T^{n+1} v\right\| \\
& +v_{n} \sup _{v \in C}\left\|T^{n+1} v-T^{n} v\right\|+\omega_{n+1}\left(\left\|v_{n+1}-v_{n}\right\|+\left|\beta_{n+1}-\beta_{n}\right|\left\|\delta g\left(v_{n}\right)-\bar{V} L v_{n}\right\|\right) \\
& +\left|\omega_{n+1}-\omega_{n}\right| \| Q_{C}(I-\lambda \widehat{B}) u_{n} \mid \\
& \leq\left(\mu_{n+1}+v_{n+1} k_{n+1} \xi_{n}+\omega_{n+1}\right)\left\|v_{n+1}-v_{n}\right\|+v_{n+1} k_{n+1}\left(1-\xi_{n+1}\right)\left\|v_{n+2}-v_{n+1}\right\| \\
& +\left|\mu_{n+1}-\mu_{n}\right|\left\|v_{n}\right\|+\left|v_{n+1}-v_{n}\right|\left\|T^{n+1} v||+v_{n} \sup _{v \in C}\right\| T^{n+1} v-T^{n} v \| \\
& +\omega_{n+1}\left|\beta_{n+1}-\beta_{n}\right||| \delta g\left(v_{n}\right)-\bar{V} L v_{n}\left\|+\left|\omega_{n+1}-\omega_{n}\right|\right\| Q_{C}(I-\lambda \widehat{B}) u_{n} \| .
\end{aligned}
$$

Which implies that

$$
\begin{aligned}
& {\left[1-v_{n+1} k_{n+1}\left(1-\xi_{n+1}\right)\right]\left\|v_{n+2}-v_{n+1}\right\|} \\
& \quad \leq\left(\mu_{n+1}+v_{n+1} k_{n+1} \xi_{n}+\omega_{n+1}\right)\left\|v_{n+1}-v_{n}\right\|+\left|\mu_{n+1}-\mu_{n}\right|\left\|v_{n}\right\|+\left|v_{n+1}-v_{n}\right|\left\|T^{n+1} v\right\| \\
& \quad+v_{n} \sup _{v \in C}\left\|T^{n+1} v-T^{n} v\right\|+\omega_{n+1}\left|\beta_{n+1}-\beta_{n}\right|\left\|\delta g\left(v_{n}\right)-\bar{V} L v_{n}\right\|+\left|\omega_{n+1}-\omega_{n}\right|\left\|Q_{C}(I-\lambda \widehat{B}) u_{n}\right\| .
\end{aligned}
$$


By conditions (ii)-(iv) in Theorem 1, we have

$$
\begin{aligned}
\| v_{n+2} & -v_{n+1} \| \\
\leq & \frac{\mu_{n+1}+v_{n+1} k_{n+1} \xi_{n}+\omega_{n+1}}{1-v_{n+1} k_{n+1}\left(1-\xi_{n+1}\right)}\left\|v_{n+1}-v_{n}\right\|+\frac{\left|\mu_{n+1}-\mu_{n}\right|}{1-v_{n+1} k_{n+1}\left(1-\xi_{n+1}\right)}\left\|v_{n}\right\| \\
& +\frac{\left|v_{n+1}-v_{n}\right|}{1-v_{n+1} k_{n+1}\left(1-\xi_{n+1}\right)}\left\|T^{n+1} v\right\|+\frac{v_{n}}{1-v_{n+1} k_{n+1}\left(1-\xi_{n+1}\right)} \sup _{v \in C} \| T^{n+1} v-T^{n_{v} \|} \\
& +\frac{\omega_{n+1}\left|\beta_{n+1}-\beta_{n}\right|}{1-v_{n+1} k_{n+1}\left(1-\xi_{n+1}\right)}\left\|\delta g\left(v_{n}\right)-\bar{V} L v_{n}\right\|+\frac{\left|\omega_{n+1}-\omega_{n}\right|}{1-v_{n+1} k_{n+1}\left(1-\xi_{n+1}\right)}\left\|Q_{C}(I-\lambda \widehat{B}) u_{n}\right\| \\
\leq & {\left[1+\frac{v_{n+1}\left(k_{n+1}-1\right)-v_{n+1} k_{n+1}\left(\xi_{n+1}-\xi_{n}\right)}{1-v_{n+1} k_{n+1}\left(1-\xi_{n+1}\right)} \mid\left\|v_{n+1}-v_{n}\right\|+\frac{\left|\mu_{n+1}-\mu_{n}\right|}{1-v_{n+1} k_{n+1}\left(1-\xi_{n+1}\right)}\left\|v_{n}\right\|\right.} \\
& +\frac{\left|v_{n+1}-v_{n}\right|}{1-v_{n+1} k_{n+1}\left(1-\xi_{n+1}\right)}\left\|T^{n+1} v\right\|+\frac{v_{n}}{1-v_{n+1} k_{n+1}\left(1-\xi_{n+1}\right)} \sup _{v \in C}\left\|T^{n+1} v-T^{n}\right\| \\
& +\frac{\omega_{n+1}\left|\beta_{n+1}-\beta_{n}\right|}{1-v_{n+1} k_{n+1}\left(1-\xi_{n+1}\right)}\left\|\delta g\left(v_{n}\right)-\bar{V} L v_{n}\right\|+\frac{\left|\omega_{n+1}-\omega_{n}\right|}{1-v_{n+1} k_{n+1}\left(1-\xi_{n+1}\right)}\left\|Q_{C}(I-\lambda \widehat{B}) u_{n}\right\| \\
\leq & {\left[1+\frac{v_{n+1}\left(k_{n+1}-1\right)}{\tau}\right]\left\|v_{n+1}-v_{n}\right\|+\frac{\omega_{n+1}\left|\beta_{n+1}-\beta_{n}\right|}{\tau}\left\|\delta g\left(v_{n}\right)-\bar{V} L v_{n}\right\| } \\
& +\frac{\left|\mu_{n+1}-\mu_{n}\right|}{\tau}\left\|v_{n}\right\|+\frac{\left|v_{n+1}-v_{n}\right|}{\tau}\left\|T^{n+1} v\right\|+\frac{v_{n}}{\tau} \sup _{v \in C}\left\|T^{n+1} v-T_{v}^{n}\right\| \\
& +\frac{\left|\omega_{n+1}-\omega_{n}\right|}{\tau}\left\|Q_{C}(I-\lambda \widehat{B}) u_{n}\right\| \\
\leq & \left(1+\varepsilon \beta_{n+1}\right)\left\|v_{n+1}-v_{n}\right\|+M_{n 1},
\end{aligned}
$$

where

$$
\begin{aligned}
M_{n_{1}} & =\frac{\left|\mu_{n+1}-\mu_{n}\right|}{\tau}\left\|v_{n}\right\|+\frac{\left|v_{n+1}-v_{n}\right|}{\tau}\left\|T^{n+1} v\right\|+\frac{v_{n}}{\tau} \sup _{v \in C}\left\|T^{n+1} v-T^{n} v\right\| \\
& +\frac{\omega_{n+1}\left|\beta_{n+1}-\beta_{n}\right|}{\tau}\left\|\delta g\left(v_{n}\right)-\bar{V} L v_{n}\right\|+\frac{\left|\omega_{n+1}-\omega_{n}\right|}{\tau}\left\|Q_{C}(I-\lambda \widehat{B}) u_{n}\right\| .
\end{aligned}
$$

We set

$$
v_{n+1}=\left(1-\mu_{n}\right) z_{n}+\mu_{n} v_{n}
$$

where

$$
z_{n}=\frac{v_{n+1}-\mu_{n} v_{n}}{1-\mu_{n}}, \forall n \geq 0
$$

Since

$$
v_{n+1}-\mu_{n} v_{n}=v_{n} T^{n}\left(\xi_{n} v_{n}+\left(1-\xi_{n}\right) v_{n+1}\right)+\omega_{n} Q_{C}(I-\lambda \widehat{B}) u_{n},
$$


we attain

$$
\begin{aligned}
& z_{n+1}-z_{n} \\
& =\frac{v_{n+2}-\mu_{n} v_{n+1}}{1-\mu_{n+1}}-\frac{v_{n+1}-\mu_{n} v_{n}}{1-\mu_{n}} \\
& =\frac{v_{n+1} T^{n+1}\left(\xi_{n+1} v_{n+1}+\left(1-\xi_{n+1}\right) v_{n+2}\right)+\omega_{n+1} Q_{C}(I-\lambda \widehat{B}) u_{n+1}}{1-\mu_{n+1}} \\
& -\frac{v_{n} T^{n}\left(\xi_{n} v_{n}+\left(1-\xi_{n}\right) v_{n+1}\right)+\omega_{n} Q_{C}(I-\lambda \widehat{B}) u_{n}}{1-\mu_{n}} \\
& =\frac{v_{n+1}}{1-\mu_{n+1}} T^{n+1}\left(\xi_{n+1} v_{n+1}+\left(1-\xi_{n+1}\right) v_{n+2}\right)-\frac{v_{n}}{1-\mu_{n}} T^{n}\left(\xi_{n} v_{n}+\left(1-\xi_{n}\right) v_{n+1}\right) \\
& +\frac{\omega_{n+1}}{1-\mu_{n+1}} Q_{C}(I-\lambda \widehat{B}) u_{n+1}-\frac{\omega_{n}}{1-\mu_{n}} Q_{C}(I-\lambda \widehat{B}) u_{n} \\
& =\frac{v_{n+1}}{1-\mu_{n+1}} T^{n+1}\left(\xi_{n+1} v_{n+1}+\left(1-\xi_{n+1}\right) v_{n+2}\right)-\frac{v_{n+1}}{1-\mu_{n+1}} T^{n+1}\left(\xi_{n} v_{n}+\left(1-\xi_{n}\right) v_{n+1}\right) \\
& +\frac{v_{n+1}}{1-\mu_{n+1}} T^{n+1}\left(\xi_{n} v_{n}+\left(1-\xi_{n}\right) v_{n+1}\right)-\frac{v_{n}}{1-\mu_{n}} T^{n+1}\left(\xi_{n} v_{n}+\left(1-\xi_{n}\right) v_{n+1}\right) \\
& +\frac{v_{n}}{1-\mu_{n}} T^{n+1}\left(\xi_{n} v_{n}+\left(1-\xi_{n}\right) v_{n+1}\right)-\frac{v_{n}}{1-\mu_{n}} T^{n}\left(\xi_{n} v_{n}+\left(1-\xi_{n}\right) v_{n+1}\right) \\
& +\frac{\omega_{n+1}}{1-\mu_{n+1}} Q_{C}(I-\lambda \widehat{B}) u_{n+1}-\frac{\omega_{n+1}}{1-\mu_{n+1}} Q_{C}(I-\lambda \widehat{B}) u_{n}+\frac{\omega_{n+1}}{1-\mu_{n+1}} Q_{C}(I-\lambda \widehat{B}) u_{n} \\
& -\frac{\omega_{n}}{1-\mu_{n}} Q_{C}(I-\lambda \widehat{B}) u_{n} \\
& =\frac{v_{n+1}}{1-\mu_{n+1}}\left(T^{n+1}\left(\xi_{n+1} v_{n+1}+\left(1-\xi_{n+1}\right) v_{n+2}\right)-T^{n+1}\left(\xi_{n} v_{n}+\left(1-\xi_{n}\right) v_{n+1}\right)\right) \\
& +\left(\frac{v_{n+1}}{1-\mu_{n+1}}-\frac{v_{n}}{1-\mu_{n}}\right) T^{n+1}\left(\xi_{n} v_{n}+\left(1-\xi_{n}\right) v_{n+1}\right)+\frac{v_{n}}{1-\mu_{n}}\left(T^{n+1}\left(\xi_{n} v_{n}+\left(1-\xi_{n}\right) v_{n+1}\right)\right. \\
& \left.-T^{n}\left(\xi_{n} v_{n}+\left(1-\xi_{n}\right) v_{n+1}\right)\right)+\frac{\omega_{n+1}}{1-\mu_{n+1}}\left(Q_{C}(I-\lambda \widehat{B}) u_{n+1}-Q_{C}(I-\lambda \widehat{B}) u_{n}\right) \\
& +\left(\frac{\omega_{n+1}}{1-\mu_{n+1}}-\frac{\omega_{n}}{1-\mu_{n}}\right) Q_{C}(I-\lambda \widehat{B}) u_{n}
\end{aligned}
$$

Thus, we acquire

$$
\begin{aligned}
& \left\|z_{n+1}-z_{n}\right\| \\
& \leq \quad \frac{v_{n+1}}{1-\mu_{n+1}}\left\|T^{n+1}\left(\xi_{n+1} v_{n+1}+\left(1-\xi_{n+1}\right) v_{n+2}\right)-T^{n+1}\left(\xi_{n} v_{n}+\left(1-\xi_{n}\right) v_{n+1}\right)\right\| \\
& \quad+\left|\frac{v_{n+1}}{1-\mu_{n+1}}-\frac{v_{n}}{1-\mu_{n}}\right|\left\|T^{n+1} v\right\|+\frac{v_{n}}{1-\mu_{n}}\left\|T^{n+1} v-T^{n} v\right\|+\frac{\omega_{n+1}}{1-\mu_{n+1}} \| Q_{C}(I-\lambda \widehat{B}) u_{n+1} \\
& \quad-Q_{C}(I-\lambda \widehat{B}) u_{n}\left\|+\left|\frac{\omega_{n+1}}{1-\mu_{n+1}}-\frac{\omega_{n}}{1-\mu_{n}}\right|\right\| Q_{C}(I-\lambda \widehat{B}) u_{n} \|
\end{aligned}
$$




$$
\begin{aligned}
& \leq \frac{v_{n+1}}{1-\mu_{n+1}} k_{n+1}\left\|\xi_{n+1} v_{n+1}+\left(1-\xi_{n+1}\right) v_{n+2}-\left[\xi_{n} v_{n}+\left(1-\xi_{n}\right) v_{n+1}\right]\right\|+\mid \frac{v_{n+1}}{1-\mu_{n+1}} \\
& -\frac{v_{n}}{1-\mu_{n}} \mid\left\|T^{n+1} v\right\|+\frac{v_{n}}{1-\mu_{n}}\left\|T^{n+1} v-T^{n} v\right\|+\frac{\omega_{n+1}}{1-\mu_{n+1}}\left\|u_{n+1}-u_{n}\right\| \\
& +\left|\frac{\omega_{n+1}}{1-\mu_{n+1}}-\frac{\omega_{n}}{1-\mu_{n}}\right|\left\|Q_{C}(I-\lambda \widehat{B}) u_{n}\right\| \\
& \leq \frac{v_{n+1}}{1-\mu_{n+1}} k_{n+1}\left\|\xi_{n}\left(v_{n+1}-v_{n}\right)+\left(1-\xi_{n+1}\right)\left(v_{n+2}-v_{n+1}\right)\right\|+\mid \frac{v_{n+1}}{1-\mu_{n+1}}-\frac{v_{n}}{1-\mu_{n+1}} \\
& +\frac{v_{n}}{1-\mu_{n+1}}-\frac{v_{n}}{1-\mu_{n}} \mid\left\|T^{n+1} v\right\|+\frac{v_{n}}{1-\mu_{n}} \sup _{v \in C}\left\|T^{n+1} v-T^{n} v\right\|+\frac{\omega_{n+1}}{1-\mu_{n+1}}\left\|u_{n+1}-u_{n}\right\| \\
& +\left|\frac{\omega_{n+1}}{1-\mu_{n+1}}-\frac{\omega_{n}}{1-\mu_{n+1}}+\frac{\omega_{n}}{1-\mu_{n+1}}-\frac{\omega_{n}}{1-\mu_{n}}\right| \| Q_{C}(I-\lambda \widehat{B}) u_{n}|| \\
& \leq \frac{v_{n+1} k_{n+1} \xi_{n}}{1-\mu_{n+1}}\left\|v_{n+1}-v_{n}\right\|+\frac{v_{n+1} k_{n+1}\left(1-\xi_{n+1}\right)}{1-\mu_{n+1}}\left\|v_{n+2}-v_{n+1}\right\|+\frac{\left|v_{n+1}-v_{n}\right|}{1-\mu_{n+1}}\left\|T^{n+1} v\right\| \\
& +v_{n} \frac{\left|\mu_{n+1}-\mu_{n}\right|}{\left(1-\mu_{n+1}\right)\left(1-\mu_{n}\right)}\left\|T^{n+1} v\right\|+\frac{v_{n}}{1-\mu_{n}} \sup _{v \in C}\left\|T^{n+1} v-T^{n} v\right\|+\frac{\omega_{n+1}}{1-\mu_{n+1}}\left\|u_{n+1}-u_{n}\right\| \\
& +\frac{\left|\omega_{n+1}-\omega_{n}\right|}{1-\mu_{n+1}}\left\|Q_{C}(I-\lambda \widehat{B}) u_{n}\right\|+\omega_{n} \frac{\left|\mu_{n+1}-\mu_{n}\right|}{\left(1-\mu_{n+1}\right)\left(1-\mu_{n}\right)}\left\|Q_{C}(I-\lambda \widehat{B}) u_{n}\right\| \\
& \leq \frac{v_{n+1} k_{n+1} \xi_{n}}{1-\mu_{n+1}}\left\|v_{n+1}-v_{n}\right\|+\frac{v_{n+1} k_{n+1}\left(1-\xi_{n+1}\right)}{1-\mu_{n+1}}\left\|v_{n+2}-v_{n+1}\right\|+\frac{\left|v_{n+1}-v_{n}\right|}{1-\mu_{n+1}}\left\|T^{n+1} v\right\| \\
& +\frac{\left|\mu_{n+1}-\mu_{n}\right|}{\left(1-\mu_{n+1}\right)\left(1-\mu_{n}\right)}\left\|T^{n+1} v\right\|+\frac{v_{n}}{1-\mu_{n}} \sup _{v \in C}\left\|T^{n+1} v-T^{n} v\right\|+\frac{\omega_{n+1}}{1-\mu_{n+1}}\left\|u_{n+1}-u_{n}\right\| \\
& +\frac{\left|\omega_{n+1}-\omega_{n}\right|}{1-\mu_{n+1}}\left\|Q_{C}(I-\lambda \widehat{B}) u_{n}\right\|+\frac{\left|\mu_{n+1}-\mu_{n}\right|}{\left(1-\mu_{n+1}\right)\left(1-\mu_{n}\right)}\left\|Q_{C}(I-\lambda \widehat{B}) u_{n}\right\| .
\end{aligned}
$$

From (7), (8) and condition (ii), (iii) in Theorem 1, we can compute

$$
\begin{aligned}
\| z_{n+1} & -z_{n} \| \\
\leq & \frac{v_{n+1} k_{n+1} \xi_{n}}{1-\mu_{n+1}}\left\|v_{n+1}-v_{n}\right\|+\frac{v_{n+1} k_{n+1}\left(1-\xi_{n+1}\right)}{1-\mu_{n+1}}\left[\left(1+\varepsilon \beta_{n+1}\right)\left\|v_{n+1}-v_{n}\right\|+M_{n_{1}}\right] \\
& +\frac{\left|v_{n+1}-v_{n}\right|}{1-\mu_{n+1}}\left\|T^{n+1} v\right\|+\frac{\left|\mu_{n+1}-\mu_{n}\right|}{\left(1-\mu_{n+1}\right)\left(1-\mu_{n}\right)}\left\|T^{n+1} v\right\|+\frac{v_{n}}{1-\mu_{n}} \sup _{v \in C}\left\|T^{n+1} v-T^{n} v\right\| \\
& +\frac{\omega_{n+1}}{1-\mu_{n+1}}\left(\left\|v_{n+1}-v_{n}\right\|+\left|\beta_{n+1}-\beta_{n}\right|\left\|\delta g\left(v_{n}\right)-\bar{V} L v_{n}\right\|\right)+\frac{\left|\omega_{n+1}-\omega_{n}\right|}{1-\mu_{n+1}}\left\|Q_{C}(I-\lambda \widehat{B}) u_{n}\right\| \\
& +\frac{\left|\mu_{n+1}-\mu_{n}\right|}{\left(1-\mu_{n+1}\right)\left(1-\mu_{n}\right)}\left\|Q_{C}(I-\lambda \widehat{B}) u_{n}\right\| \\
\leq & {\left[\frac{v_{n+1} k_{n+1} \xi_{n}}{1-\mu_{n+1}}+\frac{v_{n+1} k_{n+1}\left(1-\xi_{n+1}\right)}{1-\mu_{n+1}}\left(1+\varepsilon \beta_{n+1}\right)+\frac{\omega_{n+1}}{1-\mu_{n+1}}\right]\left\|v_{n+1}-v_{n}\right\| } \\
& +\frac{v_{n+1} k_{n+1}\left(1-\xi_{n+1}\right)}{1-\mu_{n+1}} M_{n_{1}}+\frac{\left|v_{n+1}-v_{n}\right|}{1-h_{2}}\left\|T^{n+1} v\right\|+\frac{\left|\mu_{n+1}-\mu_{n}\right|}{\left(1-h_{2}\right)^{2}}\left\|T^{n+1} v\right\| \\
& +\frac{v_{n}}{1-h_{2}} \sup _{v \in C}\left\|T^{n+1} v-T^{n} v\right\|+\frac{\omega_{n+1}\left|\beta_{n+1}-\beta_{n}\right|}{1-h_{2}}\left\|\delta g\left(v_{n}\right)-\bar{V} L v_{n}\right\| \\
& +\frac{\left|\omega_{n+1}-\omega_{n}\right|}{1-h_{2}}\left\|Q_{C}(I-\lambda \widehat{B}) u_{n}\right\|+\frac{\left|\mu_{n+1}-\mu_{n}\right|}{\left(1-h_{2}\right)^{2}}\left\|Q_{C}(I-\lambda \widehat{B}) u_{n}\right\| \\
\leq & {\left[\frac{\left.v_{n+1} k_{n+1} \xi_{n}+v_{n+1} k_{n+1}\left(1-\xi_{n+1}\right)+\omega_{n+1}+\frac{v_{n+1} k_{n+1}\left(1-\xi_{n+1}\right) \varepsilon \beta_{n+1}}{1-\mu_{n+1}}\right]\left\|v_{n+1}-v_{n}\right\|}{1-\mu_{n+1}}\right.} \\
& +\frac{v_{n+1} k_{n+1}\left(1-\xi_{n+1}\right)}{1-\mu_{n+1}} M_{n_{1}}+M_{n_{2}} \\
\leq & {\left[1+\frac{v_{n+1}\left(k_{n+1}\left(\xi_{n}+1-\xi_{n+1}\right)-1\right)}{1-\mu_{n+1} k_{n+1}\left(1-\xi_{n+1}\right) \varepsilon \beta_{n+1}}\right]\left\|v_{n+1}-v_{n}\right\| } \\
&
\end{aligned}
$$




$$
\begin{aligned}
& +\frac{v_{n+1} k_{n+1}\left(1-\xi_{n+1}\right)}{1-\mu_{n+1}} M_{n_{1}}+M_{n_{2}} \\
\leq & {\left[1+\frac{v_{n+1}\left(k_{n+1}-1\right)}{1-\mu_{n+1}}+\frac{v_{n+1} k_{n+1}\left(1-\xi_{n+1}\right) \varepsilon \beta_{n+1}}{1-\mu_{n+1}}\right]\left\|v_{n+1}-v_{n}\right\| } \\
& +\frac{v_{n+1} k_{n+1}\left(1-\xi_{n+1}\right)}{1-\mu_{n+1}} M_{n_{1}}+M_{n_{2}} \\
\leq & {\left[1+\frac{v_{n+1} \varepsilon \beta_{n+1}}{1-\mu_{n+1}}+\frac{v_{n+1} k_{n+1} \varepsilon \beta_{n+1}}{1-\mu_{n+1}}\right]\left\|v_{n+1}-v_{n}\right\|+\frac{v_{n+1} k_{n+1}\left(1-\xi_{n+1}\right)}{1-\mu_{n+1}} M_{n_{1}}+M_{n_{2}} } \\
\leq & {\left[1+\frac{\left(k_{n+1}+1\right) v_{n+1} \varepsilon \beta_{n+1}}{1-h_{2}}\right]\left\|v_{n+1}-v_{n}\right\|+\frac{v_{n+1} k_{n+1}\left(1-\xi_{n+1}\right)}{1-h_{2}} M_{n_{1}}+M_{n_{2},} }
\end{aligned}
$$

where

$$
\begin{aligned}
M_{n_{2}} & =\frac{\left|v_{n+1}-v_{n}\right|}{1-h_{2}}\left\|T^{n+1} v\right\|+\frac{\left|\mu_{n+1}-\mu_{n}\right|}{\left(1-h_{2}\right)^{2}}\left\|T^{n+1} v\right\| \\
& +\frac{v_{n}}{1-h_{2}} \sup _{x \in C}\left\|T^{n+1} v-T^{n} v\right\|+\frac{\omega_{n+1}\left|\beta_{n+1}-\beta_{n}\right|}{1-h_{2}}\left\|\delta g\left(v_{n}\right)-\bar{V} L v_{n}\right\| \\
& +\frac{\left|\omega_{n+1}-\omega_{n}\right|}{1-h_{2}}\left\|Q_{C}(I-\lambda \widehat{B}) u_{n}\right\|+\frac{\left|\mu_{n+1}-\mu_{n}\right|}{\left(1-h_{2}\right)^{2}}\left\|Q_{C}(I-\lambda \widehat{B}) u_{n}\right\| .
\end{aligned}
$$

So, by conditions $(i)$ and (ii) in Theorem 1 , we gain

$$
\limsup _{n \rightarrow \infty}\left(\left\|z_{n+1}-z_{n}\right\|-\left\|v_{n+1}-v_{n}\right\|\right) \leq 0 .
$$

Adopting Lemma 3, we gain

$$
\lim _{n \rightarrow \infty}\left\|z_{n}-v_{n}\right\|=0
$$

Taking notice of $v_{n+1}-v_{n}=\left(1-\mu_{n}\right)\left(z_{n}-v_{n}\right)$. Then

$$
\lim _{n \rightarrow \infty}\left\|v_{n+1}-v_{n}\right\|=0
$$

From (7), (9) and condition ( $i$ ) in Theorem 1, we receive

$$
\lim _{n \rightarrow \infty}\left\|u_{n+1}-u_{n}\right\|=0
$$

Step 4: Again by the definition of $u_{n}$ and Lemma 4, we get

$$
\begin{aligned}
\left\|u_{n}-q\right\|^{2} & =\left\|\beta_{n} \delta g\left(v_{n}\right)+\left(I-\beta_{n} \bar{V}\right) L v_{n}-q\right\|^{2} \\
& =\left\|\left(L v_{n}-q\right)+\left(\beta_{n} \delta g\left(v_{n}\right)-\beta_{n} \bar{V} L v_{n}\right)\right\|^{2} \\
& \leq\left\|L v_{n}-q\right\|^{2}+2 \beta_{n}\left\langle\delta g\left(v_{n}\right)-\bar{V} L v_{n}, j\left(u_{n}-q\right)\right\rangle \\
& \leq\left\|v_{n}-q\right\|^{2}+2 \beta_{n}\left\|\delta g\left(v_{n}\right)-\bar{V} L v_{n}\right\|\left\|u_{n}-q\right\| .
\end{aligned}
$$

By the non-expansiveness of $Q_{C}$, Lemma 7 and (10), we have

$$
\begin{aligned}
\left\|v_{n+1}-q\right\|^{2}= & \left\|\mu_{n} v_{n}+v_{n} T^{n}\left(\xi_{n} v_{n}+\left(1-\xi_{n}\right) v_{n+1}\right)+\omega_{n} Q_{C}(I-\lambda \widehat{B}) u_{n}-q\right\|^{2} \\
\leq & \mu_{n}\left\|v_{n}-q\right\|^{2}+v_{n}\left\|T^{n}\left(\xi_{n} v_{n}+\left(1-\xi_{n}\right) v_{n+1}\right)-q\right\|^{2}+\omega_{n}\left\|Q_{C}(I-\lambda \widehat{B}) u_{n}-q\right\|^{2} \\
\leq & \mu_{n}\left\|v_{n}-q\right\|^{2}+v_{n} k_{n}^{2}\left\|\left(\xi_{n} v_{n}+\left(1-\xi_{n}\right) v_{n+1}\right)-q\right\|^{2}+\omega_{n}\left\|(I-\lambda \widehat{B}) u_{n}-(I-\lambda \widehat{B}) q\right\|^{2} \\
\leq & \mu_{n}\left\|v_{n}-q\right\|^{2}+v_{n} k_{n}^{2} \xi_{n}\left\|v_{n}-q\right\|^{2}+v_{n} k_{n}^{2}\left(1-\xi_{n}\right)\left\|v_{n+1}-q\right\|^{2}+\omega_{n}\left\|u_{n}-q\right\|^{2} \\
& -\omega_{n} \lambda(2 \widehat{b}-\lambda)\left\|\widehat{B} u_{n}-\widehat{B} q\right\|^{2}
\end{aligned}
$$




$$
\begin{aligned}
\leq & \mu_{n}\left\|v_{n}-q\right\|^{2}+v_{n} k_{n}^{2} \xi_{n}\left\|v_{n}-q\right\|^{2}+v_{n} k_{n}^{2}\left(1-\xi_{n}\right)\left\|v_{n+1}-q\right\|^{2}+\omega_{n}\left\|v_{n}-q\right\|^{2} \\
& +2 \omega_{n} \beta_{n}\left\|\delta g\left(v_{n}\right)-\bar{V} L v_{n}\right\|\left\|u_{n}-q\right\|-\omega_{n} \lambda(2 \widehat{b}-\lambda)\left\|\widehat{B} u_{n}-\widehat{B} q\right\|^{2} \\
\leq & \left(\mu_{n}+v_{n} k_{n}^{2} \xi_{n}+\omega_{n}\right)\left\|v_{n}-q\right\|^{2}+v_{n} k_{n}^{2}\left(1-\xi_{n}\right)\left\|v_{n+1}-q\right\|^{2}+2 \omega_{n} \beta_{n} \| \delta g\left(v_{n}\right) \\
& -\bar{V} L v_{n}\|\| u_{n}-q\left\|-\omega_{n} \lambda(2 \widehat{b}-\lambda)\right\| \widehat{B} u_{n}-\widehat{B} q \|^{2} .
\end{aligned}
$$

This implies that

$$
\begin{aligned}
& \omega_{n} \lambda(2 \widehat{b}-\lambda)\left\|\widehat{B} u_{n}-\widehat{B} q\right\|^{2} \leq\left(\mu_{n}+v_{n} k_{n}^{2} \xi_{n}+\omega_{n}\right)\left\|v_{n}-q\right\|^{2}-\left[1-v_{n} k_{n}^{2}\left(1-\xi_{n}\right)\right]\left\|v_{n+1}-q\right\|^{2} \\
& \quad+2 \omega_{n} \beta_{n}\left\|\delta g\left(v_{n}\right)-\bar{V} L v_{n}\right\|\left\|u_{n}-q\right\| \\
& \leq\left(1-v_{n}+v_{n} k_{n}^{2} \xi_{n}\right)\left\|v_{n}-q\right\|^{2}-\left[1-v_{n} k_{n}^{2}\left(1-\xi_{n}\right)\right]\left\|v_{n+1}-q\right\|^{2}+2 \omega_{n} \beta_{n}\left\|\delta g\left(v_{n}\right)-\bar{V} L v_{n}\right\|\left\|u_{n}-q\right\| \\
& \leq\left(1-v_{n} k_{n}^{2}+v_{n} k_{n}^{2} \xi_{n}+v_{n} k_{n}^{2}-v_{n}\right)\left\|v_{n}-q\right\|^{2}-\left[1-v_{n} k_{n}^{2}\left(1-\xi_{n}\right)\right]\left\|v_{n+1}-q\right\|^{2} \\
&+2 \omega_{n} \beta_{n}\left\|\delta g\left(v_{n}\right)-\bar{V} L v_{n}\right\|\left\|u_{n}-q\right\| \\
& \leq\left(1-v_{n} k_{n}^{2}\left(1-\xi_{n}\right)\right)\left[\left\|v_{n}-q\right\|^{2}-\left\|v_{n+1}-q\right\|^{2}\right]+2 \omega_{n} \beta_{n}\left\|\delta g\left(v_{n}\right)-\bar{V} L v_{n}\right\|\left\|u_{n}-q\right\| \\
&+v_{n}\left(k_{n}^{2}-1\right)\left\|v_{n}-q\right\|^{2} \\
& \leq\left(\left\|v_{n}-q\right\|+\left\|v_{n+1}-q\right\|\right)\left\|v_{n}-v_{n+1}\right\|+2 \omega_{n} \beta_{n}\left\|\delta g\left(v_{n}\right)-\bar{V} L v_{n}\right\|\left\|u_{n}-q\right\|+v_{n} \varepsilon \beta_{n}\left\|v_{n}-q\right\|^{2} .
\end{aligned}
$$

By conditions ( $i)$, (ii) in Theorem 1 and (9), we can acquire

$$
\lim _{n \rightarrow \infty}\left\|\widehat{B} u_{n}-\widehat{B} q\right\|=0 .
$$

Applying Lemma 2 and (10), we obtain

$$
\begin{aligned}
\left\|v_{n+1}-q\right\|^{2}= & \left\|\mu_{n} v_{n}+v_{n} T^{n}\left(\xi_{n} v_{n}+\left(1-\xi_{n}\right) v_{n+1}\right)+\omega_{n} Q_{C}(I-\lambda \widehat{B}) u_{n}-q\right\|^{2} \\
\leq & \mu_{n}\left\|v_{n}-q\right\|^{2}+v_{n}\left\|T^{n}\left(\xi_{n} v_{n}+\left(1-\xi_{n}\right) v_{n+1}\right)-q\right\|^{2}+\omega_{n}\left\|Q_{C}(I-\lambda \widehat{B}) u_{n}-q\right\|^{2} \\
& -\mu_{n} v_{n} m_{1}\left(\left\|v_{n}-T^{n}\left(\xi_{n} v_{n}+\left(1-\xi_{n}\right) v_{n+1}\right)\right\|\right)-\mu_{n} \omega_{n} m_{1}\left(\left\|v_{n}-Q_{C}(I-\lambda \widehat{B}) u_{n}\right\|\right) \\
\leq & \mu_{n}\left\|v_{n}-q\right\|^{2}+v_{n} k_{n}^{2}\left\|\left(\xi_{n} v_{n}+\left(1-\xi_{n}\right) v_{n+1}\right)-q\right\|^{2}+\omega_{n}\left\|u_{n}-q\right\|^{2} \\
& -\mu_{n} v_{n} m_{1}\left(\left\|v_{n}-T^{n}\left(\xi_{n} v_{n}+\left(1-\xi_{n}\right) v_{n+1}\right)\right\|\right)-\mu_{n} \omega_{n} m_{1}\left(\left\|v_{n}-Q_{C}(I-\lambda \widehat{B}) u_{n}\right\|\right) \\
\leq & \mu_{n}\left\|v_{n}-q\right\|^{2}+v_{n} k_{n}^{2} \xi_{n}\left\|v_{n}-q\right\|^{2}+v_{n} k_{n}^{2}\left(1-\xi_{n}\right)\left\|v_{n+1}-q\right\|^{2}+\omega_{n}\left\|u_{n}-q\right\|^{2} \\
& -\mu_{n} v_{n} m_{1}\left(\left\|v_{n}-T^{n}\left(\xi_{n} v_{n}+\left(1-\xi_{n}\right) v_{n+1}\right)\right\|\right)-\mu_{n} \omega_{n} m_{1}\left(\left\|v_{n}-Q_{C}(I-\lambda \widehat{B}) u_{n}\right\|\right) \\
\leq & \left(\mu_{n}+v_{n} k_{n}^{2} \xi_{n}+\omega_{n}\right)\left\|v_{n}-q\right\|^{2}+v_{n} k_{n}^{2}\left(1-\xi_{n}\right)\left\|v_{n+1}-q\right\|^{2} \\
& +2 \omega_{n} \beta_{n}\left\|\delta g\left(v_{n}\right)-\bar{V} L v_{n}\right\|\left\|u_{n}-q\right\|-\mu_{n} v_{n} m_{1}\left(\left\|v_{n}-T^{n}\left(\xi_{n} v_{n}+\left(1-\xi_{n}\right) v_{n+1}\right)\right\|\right) \\
& -\mu_{n} \omega_{n} m_{1}\left(\left\|v_{n}-Q_{C}(I-\lambda \widehat{B}) u_{n}\right\|\right) .
\end{aligned}
$$

Which implies that

$$
\begin{aligned}
& \mu_{n} v_{n} m_{1}\left(\| v_{n}-T^{n}\left(\xi_{n} v_{n}+\left(1-\xi_{n}\right) v_{n+1} \|\right)+\mu_{n} \omega_{n} m_{1}\left(\left\|v_{n}-Q_{C}(I-\lambda \widehat{B}) u_{n}\right\|\right)\right. \\
& \leq\left(\mu_{n}+v_{n} k_{n}^{2} \xi_{n}+\omega_{n}\right)\left\|v_{n}-q\right\|^{2}-\left[1-v_{n} k_{n}^{2}\left(1-\xi_{n}\right)\right]\left\|v_{n+1}-q\right\|^{2} \\
&+2 \omega_{n} \beta_{n}\left\|\delta g\left(v_{n}\right)-\bar{V} L v_{n}\right\|\left\|u_{n}-q\right\| \\
& \leq\left(1-v_{n}+v_{n} k_{n}^{2} \xi_{n}\right)\left\|v_{n}-q\right\|^{2}-\left[1-v_{n} k_{n}^{2}\left(1-\xi_{n}\right)\right]\left\|v_{n+1}-q\right\|^{2} \\
&+2 \omega_{n} \beta_{n}\left\|\delta g\left(v_{n}\right)-\bar{V} L v_{n}\right\|\left\|u_{n}-q\right\| \\
& \leq\left(1-v_{n} k_{n}^{2}+v_{n} k_{n}^{2} \xi_{n}+v_{n} k_{n}^{2}-v_{n}\right)\left\|v_{n}-q\right\|^{2}-\left[1-v_{n} k_{n}^{2}\left(1-\xi_{n}\right)\right]\left\|v_{n+1}-q\right\|^{2} \\
&+2 \omega_{n} \beta_{n}\left\|\delta g\left(v_{n}\right)-\bar{V} L v_{n}\right\|\left\|u_{n}-q\right\| \\
& \leq {\left[1-v_{n} k_{n}^{2}\left(1-\xi_{n}\right)\right]\left(\left\|v_{n}-q\right\|^{2}-\left\|v_{n+1}-q\right\|^{2}\right)+v_{n}\left(k_{n}^{2}-1\right)\left\|v_{n}-q\right\|^{2} } \\
&+2 \omega_{n} \beta_{n}\left\|\delta g\left(v_{n}\right)-\bar{V} L v_{n}\right\|\left\|u_{n}-q\right\| \\
& \leq\left(\left\|v_{n}-q\right\|+\left\|v_{n+1}-q\right\|\right)\left\|v_{n}-v_{n+1}\right\|+v_{n} \varepsilon \beta_{n}\left\|v_{n}-q\right\|^{2}+2 \omega_{n} \beta_{n}\left\|\delta g\left(v_{n}\right)-\bar{V} L v_{n}\right\|\left\|u_{n}-q\right\| .
\end{aligned}
$$


From conditions (i), (ii) in Theorem 1 and (9), and the properties of the function $m_{1}$, we acquire

$$
\lim _{n \rightarrow \infty}\left\|v_{n}-T^{n}\left(\xi_{n} v_{n}+\left(1-\xi_{n}\right) v_{n+1}\right)\right\|=0,
$$

and

$$
\lim _{n \rightarrow \infty}\left\|v_{n}-Q_{C}(I-\lambda \widehat{B}) u_{n}\right\|=0
$$

Applying Lemma 1, Lemma 9 and (10), we gain

$$
\begin{aligned}
\left\|Q_{C}(I-\lambda \widehat{B}) u_{n}-q\right\|^{2} \leq & \left\langle(I-\lambda \widehat{B}) u_{n}-(I-\lambda \widehat{B}) q, j\left(Q_{C}(I-\lambda \widehat{B}) u_{n}-q\right)\right\rangle \\
\leq & \left\langle u_{n}-q, j\left(Q_{C}(I-\lambda \widehat{B}) u_{n}-q\right)\right\rangle-\lambda\left\langle\widehat{B} u_{n}-\widehat{B} q, j\left(Q_{C}(I-\lambda \widehat{B}) u_{n}-q\right)\right\rangle \\
\leq & \frac{1}{2}\left[\left\|u_{n}-q\right\|^{2}+\left\|Q_{C}(I-\lambda \widehat{B}) u_{n}-q\right\|^{2}-m_{2}\left(\left\|u_{n}-q-\left(Q_{C}(I-\lambda \widehat{B}) u_{n}-q\right)\right\|\right)\right] \\
& -\lambda\left\|\widehat{B} u_{n}-\widehat{B} q\right\|\left\|Q_{C}(I-\lambda \widehat{B}) u_{n}-q\right\| \\
\leq & \frac{1}{2}\left[\left\|u_{n}-q\right\|^{2}+\left\|Q_{C}(I-\lambda \widehat{B}) u_{n}-q\right\|^{2}-m_{2}\left(\left\|u_{n}-Q_{C}(I-\lambda \widehat{B}) u_{n}\right\|\right)\right] \\
& -\lambda\left\|\widehat{B} u_{n}-\widehat{B} q\right\|\left\|Q_{C}(I-\lambda \widehat{B}) u_{n}-q\right\| \\
\leq & \frac{1}{2}\left[\left\|v_{n}-q\right\|^{2}+2 \beta_{n}\left\|\delta g\left(v_{n}\right)-\bar{V} L v_{n}\right\|\left\|u_{n}-q\right\|+\left\|Q_{C}(I-\lambda \widehat{B}) u_{n}-q\right\|^{2}\right. \\
& \left.-m_{2}\left(\left\|u_{n}-Q_{C}(I-\lambda \widehat{B}) u_{n}\right\|\right)\right]-\lambda\left\|\widehat{B} u_{n}-\widehat{B} q\right\|\left\|Q_{C}(I-\lambda \widehat{B}) u_{n}-q\right\| .
\end{aligned}
$$

It follows that

$$
\begin{aligned}
\left\|Q_{C}(I-\lambda \widehat{B}) u_{n}-q\right\|^{2} \leq & \left\|v_{n}-q\right\|^{2}+2 \beta_{n}\left\|\delta g\left(v_{n}\right)-\bar{V} L v_{n}\right\|\left\|u_{n}-q\right\| \\
& -m_{2}\left(\left\|u_{n}-Q_{C}(I-\lambda \widehat{B}) u_{n}\right\|\right)-2 \lambda\left\|\widehat{B} u_{n}-\widehat{B} q\right\|\left\|Q_{C}(I-\lambda \widehat{B}) u_{n}-q\right\| .
\end{aligned}
$$

Hence, we know that

$$
\begin{aligned}
\left\|v_{n+1}-q\right\|^{2}= & \left\|\mu_{n} v_{n}+v_{n} T^{n}\left(\xi_{n} v_{n}+\left(1-\xi_{n}\right) v_{n+1}\right)+\omega_{n} Q_{C}(I-\lambda \widehat{B}) u_{n}-q\right\|^{2} \\
\leq & \mu_{n}\left\|v_{n}-q\right\|^{2}+v_{n}\left\|T^{n}\left(\xi_{n} v_{n}+\left(1-\xi_{n}\right) v_{n+1}\right)-q\right\|^{2}+\omega_{n}\left\|Q_{C}(I-\lambda \widehat{B}) u_{n}-q\right\|^{2} \\
\leq & \mu_{n}\left\|v_{n}-q\right\|^{2}+v_{n} k_{n}^{2}\left\|\left(\xi_{n} v_{n}+\left(1-\xi_{n}\right) v_{n+1}\right)-q\right\|^{2}+\omega_{n}\left\|Q_{C}(I-\lambda \widehat{B}) u_{n}-q\right\|^{2} \\
\leq & \mu \mu_{n}\left\|v_{n}-q\right\|^{2}+v_{n} k_{n}^{2} \xi_{n}\left\|v_{n}-q\right\|^{2}+v_{n} k_{n}^{2}\left(1-\xi_{n}\right)\left\|v_{n+1}-q\right\|^{2} \\
& +\omega_{n}\left[\left\|v_{n}-q\right\|^{2}+2 \beta_{n}\left\|\delta g\left(v_{n}\right)-\bar{V} L v_{n}\right\|\left\|u_{n}-q\right\|\right. \\
& \left.-m_{2}\left(\left\|u_{n}-Q_{C}(I-\lambda \widehat{B}) u_{n}\right\|\right)-2 \lambda\left\|\widehat{B} u_{n}-\widehat{B} q\right\|\left\|Q_{C}(I-\lambda \widehat{B}) u_{n}-q\right\|\right] \\
\leq & \left(\mu_{n}+v_{n} k_{n}^{2} \xi_{n}+\omega_{n}\right)\left\|v_{n}-q\right\|^{2}+v_{n} k_{n}^{2}\left(1-\xi_{n}\right)\left\|v_{n+1}-q\right\|^{2} \\
& +2 \omega_{n} \beta_{n}\left\|\delta g\left(v_{n}\right)-\bar{V} L v_{n}\right\|\left\|u_{n}-q\right\|-\omega_{n} m_{2}\left(\left\|u_{n}-Q_{C}(I-\lambda \widehat{B}) u_{n}\right\|\right) \\
& -2 \lambda \omega_{n}\left\|\widehat{B} u_{n}-\widehat{B} q\right\|\left\|Q_{C}(I-\lambda \widehat{B}) u_{n}-q\right\| .
\end{aligned}
$$

Which implies that

$$
\begin{aligned}
\omega_{n} m_{2}\left(\| u_{n}-\right. & \left.Q_{C}(I-\lambda \widehat{B}) u_{n} \|\right) \leq\left(\mu_{n}+v_{n} k_{n}^{2} \xi_{n}+\omega_{n}\right)\left\|v_{n}-q\right\|^{2}-\left[1-v_{n} k_{n}^{2}\left(1-\xi_{n}\right)\right]\left\|v_{n+1}-q\right\|^{2} \\
& +2 \omega_{n} \beta_{n}\left\|\delta g\left(v_{n}\right)-\bar{V} L v_{n}\right\|\left\|u_{n}-q\right\|-2 \lambda \omega_{n}\left\|\widehat{B} u_{n}-\widehat{B} q\right\|\left\|Q_{C}(I-\lambda \widehat{B}) u_{n}-q\right\| \\
\leq & \left(1-v_{n}+v_{n} k_{n}^{2} \xi_{n}\right)\left\|v_{n}-q\right\|^{2}-\left[1-v_{n} k_{n}^{2}\left(1-\xi_{n}\right)\right]\left\|v_{n+1}-q\right\|^{2} \\
& +2 \omega_{n} \beta_{n}\left\|\delta g\left(v_{n}\right)-\bar{V} L v_{n}\right\|\left\|u_{n}-q\right\|-2 \lambda \omega_{n}\left\|\widehat{B} u_{n}-\widehat{B} q\right\|\left\|Q_{C}(I-\lambda \widehat{B}) u_{n}-q\right\| \\
\leq & \left(1-v_{n} k_{n}^{2}+v_{n} k_{n}^{2} \xi_{n}+v_{n} k_{n}^{2}-v_{n}\right)\left\|v_{n}-q\right\|^{2}-\left[1-v_{n} k_{n}^{2}\left(1-\xi_{n}\right)\right]\left\|v_{n+1}-q\right\|^{2} \\
& +2 \omega_{n} \beta_{n}\left\|\delta g\left(v_{n}\right)-\bar{V} L v_{n}\right\|\left\|u_{n}-q\right\|-2 \lambda \omega_{n}\left\|\widehat{B} u_{n}-\widehat{B} q\right\|\left\|Q_{C}(I-\lambda \widehat{B}) u_{n}-q\right\| \\
\leq & {\left[1-v_{n} k_{n}^{2}\left(1-\xi_{n}\right)\right]\left(\left\|v_{n}-q\right\|^{2}-\left\|v_{n+1}-q\right\|^{2}\right)+v_{n}\left(k_{n}^{2}-1\right)\left\|v_{n}-q\right\|^{2} }
\end{aligned}
$$




$$
\begin{aligned}
& +2 \omega_{n} \beta_{n}\left\|\delta g\left(v_{n}\right)-\bar{V} L v_{n}\right\|\left\|u_{n}-q\right\|-2 \lambda \omega_{n}\left\|\widehat{B} u_{n}-\widehat{B} q\right\|\left\|Q_{C}(I-\lambda \widehat{B}) u_{n}-q\right\| \\
\leq & {\left[1-v_{n} k_{n}^{2}\left(1-\xi_{n}\right)\right]\left(\left\|v_{n}-q\right\|+\left\|v_{n+1}-q\right\|\right)\left\|v_{n}-v_{n+1}\right\|+v_{n}\left(k_{n}^{2}-1\right)\left\|v_{n}-q\right\|^{2} } \\
& +2 \omega_{n} \beta_{n}\left\|\delta g\left(v_{n}\right)-\bar{V} L v_{n}\right\|\left\|u_{n}-q\right\|-2 \lambda \omega_{n}\left\|\widehat{B} u_{n}-\widehat{B} q\right\|\left\|Q_{C}(I-\lambda \widehat{B}) u_{n}-q\right\| \\
\leq & \left(\left\|v_{n}-q\right\|+\left\|v_{n+1}-q\right\|\right)\left\|v_{n}-v_{n+1}\right\|+v_{n} \varepsilon \beta_{n}\left\|v_{n}-q\right\|^{2} \\
& +2 \omega_{n} \beta_{n}\left\|\delta g\left(v_{n}\right)-\bar{V} L v_{n}\right\|\left\|u_{n}-q\right\|-2 \lambda \omega_{n}\left\|\widehat{B} u_{n}-\widehat{B} q\right\|\left\|Q_{C}(I-\lambda \widehat{B}) u_{n}-q\right\| .
\end{aligned}
$$

Similarly, by conditions (i), (ii) in Theorem 1 and (9), (11) and the properties of the function $m_{2}$, we attain

$$
\lim _{n \rightarrow \infty}\left\|u_{n}-Q_{C}(I-\lambda \widehat{B}) u_{n}\right\|=0
$$

Thinking

$$
\left\|v_{n}-u_{n}\right\| \leq\left\|v_{n}-Q_{C}(I-\lambda \widehat{B}) u_{n}\right\|+\left\|Q_{C}(I-\lambda \widehat{B}) u_{n}-u_{n}\right\|,
$$

and (13) and (14), we receive

$$
\lim _{n \rightarrow \infty}\left\|v_{n}-u_{n}\right\|=0
$$

Adopting condition $(i)$ in Theorem 1 , we can get

$$
\begin{aligned}
\left\|u_{n}-L v_{n}\right\| & =\left\|\beta_{n} \delta g\left(v_{n}\right)+\left(I-\beta_{n} \bar{V}\right) L v_{n}-L v_{n}\right\| \\
& =\beta_{n}\left\|\delta g\left(v_{n}\right)-\bar{V} L v_{n}\right\| \rightarrow 0, \text { as } n \rightarrow \infty .
\end{aligned}
$$

and also

$$
\left\|v_{n}-L v_{n}\right\| \leq\left\|v_{n}-u_{n}\right\|+\left\|u_{n}-L v_{n}\right\| \rightarrow 0, \text { as } n \rightarrow \infty .
$$

Moreover, from (12), (14) and (15), we have

$$
\begin{aligned}
& \left\|Q_{C}(I-\lambda \widehat{B}) u_{n}-T^{n}\left(\xi_{n} v_{n}+\left(1-\xi_{n}\right) v_{n+1}\right)\right\| \\
& =\left\|Q_{C}(I-\lambda \widehat{B}) u_{n}-u_{n}\right\|+\left\|u_{n}-T^{n}\left(\xi_{n} v_{n}+\left(1-\xi_{n}\right) v_{n+1}\right)\right\| \\
& =\left\|Q_{C}(I-\lambda \widehat{B}) u_{n}-u_{n}\right\|+\left\|u_{n}-v_{n}\right\|+\left\|v_{n}-T^{n}\left(\xi_{n} v_{n}+\left(1-\xi_{n}\right) v_{n+1}\right)\right\| \rightarrow 0 \text { as } n \rightarrow \infty .
\end{aligned}
$$

Since

$$
\begin{aligned}
\| v_{n+1} & -T^{n}\left(\xi_{n} v_{n}+\left(1-\xi_{n}\right) v_{n+1}\right) \| \\
& =\left\|\mu_{n} v_{n}+v_{n} T^{n}\left(\xi_{n} v_{n}+\left(1-\xi_{n}\right) v_{n+1}\right)+\omega_{n} Q_{C}(I-\lambda \widehat{B}) u_{n}-T^{n}\left(\xi_{n} v_{n}+\left(1-\xi_{n}\right) v_{n+1}\right)\right\| \\
& =\left\|\mu_{n}\left[v_{n}-T^{n}\left(\xi_{n} v_{n}+\left(1-\xi_{n}\right) v_{n+1}\right)\right]+\omega_{n}\left[Q_{C}(I-\lambda \widehat{B}) u_{n}-T^{n}\left(\xi_{n} v_{n}+\left(1-\xi_{n}\right) v_{n+1}\right)\right]\right\| \\
& \leq \mu_{n}\left\|v_{n}-T^{n}\left(\xi_{n} v_{n}+\left(1-\xi_{n}\right) v_{n+1}\right)\right\|+\omega_{n}\left\|Q_{C}(I-\lambda \widehat{B}) u_{n}-T^{n}\left(\xi_{n} v_{n}+\left(1-\xi_{n}\right) v_{n+1}\right)\right\| .
\end{aligned}
$$

By condition (ii) in Theorem 1 and (12), (17), we obtain

$$
\left\|v_{n+1}-T^{n}\left(\xi_{n} v_{n}+\left(1-\xi_{n}\right) v_{n+1}\right)\right\| \rightarrow 0 \text { as } n \rightarrow \infty .
$$

We know that

$$
\begin{aligned}
\| v_{n} & -T^{n} v_{n}\|=\| v_{n}-v_{n+1}+v_{n+1}-T^{n}\left(\xi_{n} v_{n}+\left(1-\xi_{n}\right) v_{n+1}\right)+T^{n}\left(\xi_{n} v_{n}+\left(1-\xi_{n}\right) v_{n+1}\right)-T^{n} v_{n} \| \\
& \leq\left\|v_{n}-v_{n+1}\right\|+\left\|v_{n+1}-T^{n}\left(\xi_{n} v_{n}+\left(1-\xi_{n}\right) v_{n+1}\right)\right\|+\left\|T^{n}\left(\xi_{n} v_{n}+\left(1-\xi_{n}\right) v_{n+1}\right)-T^{n} v_{n}\right\| \\
& \leq\left\|v_{n}-v_{n+1}\right\|+\left\|v_{n+1}-T^{n}\left(\xi_{n} v_{n}+\left(1-\xi_{n}\right) v_{n+1}\right)\right\|+k_{n}\left\|\xi_{n} v_{n}+\left(1-\xi_{n}\right) v_{n+1}-v_{n}\right\|
\end{aligned}
$$




$$
\leq\left\|v_{n}-v_{n+1}\right\|+\left\|v_{n+1}-T^{n}\left(\xi_{n} v_{n}+\left(1-\xi_{n}\right) v_{n+1}\right)\right\|+k_{n}\left(1-\xi_{n}\right)\left\|v_{n+1}-v_{n}\right\| .
$$

By (9) and (18), we acquire

$$
\left\|v_{n}-T^{n} v_{n}\right\| \rightarrow 0, \text { as } n \rightarrow \infty .
$$

Because $T$ is an asymptotically non-expansive mapping, we attain

$$
\begin{aligned}
\left\|v_{n}-T v_{n}\right\| & =\left\|v_{n}-v_{n+1}+v_{n+1}-T^{n+1} v_{n+1}+T^{n+1} v_{n+1}-T^{n+1} v_{n}+T^{n+1} v_{n}-T v_{n}\right\| \\
& \leq\left\|v_{n}-v_{n+1}\right\|+\left\|v_{n+1}-T^{n+1} v_{n+1}\right\|+\left\|T^{n+1} v_{n+1}-T^{n+1} v_{n}\right\|+\left\|T^{n+1} v_{n}-T v_{n}\right\| \\
& \leq\left\|v_{n}-v_{n+1}\right\|+\left\|v_{n+1}-T^{n+1} v_{n+1}\right\|+k_{n+1}\left\|v_{n+1}-v_{n}\right\|+k_{1}\left\|T^{n} v_{n}-v_{n}\right\| \\
& \leq\left(1+k_{n+1}\right)\left\|v_{n}-v_{n+1}\right\|+\left\|v_{n+1}-T^{n+1} v_{n+1}\right\|+k_{1}\left\|T^{n} v_{n}-v_{n}\right\| .
\end{aligned}
$$

By (9) and (19), we acquire

$$
\left\|v_{n}-T v_{n}\right\| \rightarrow 0, \text { as } n \rightarrow \infty
$$

From (15), (16) and (20), we have

$$
\begin{aligned}
\left\|u_{n}-T u_{n}\right\| & \leq\left\|u_{n}-v_{n}\right\|+\left\|v_{n}-T v_{n}\right\|+\left\|T v_{n}-T u_{n}\right\| \\
& \leq\left\|u_{n}-v_{n}\right\|+\left\|v_{n}-T v_{n}\right\|+k_{1}\left\|v_{n}-u_{n}\right\| \rightarrow 0, \text { as } n \rightarrow \infty,
\end{aligned}
$$

and

$$
\begin{aligned}
\left\|u_{n}-L u_{n}\right\| & \leq\left\|u_{n}-v_{n}\right\|+\left\|v_{n}-L v_{n}\right\|+\left\|L v_{n}-L u_{n}\right\| \\
& \leq\left\|u_{n}-v_{n}\right\|+\left\|v_{n}-L v_{n}\right\|+\left\|v_{n}-u_{n}\right\| \rightarrow 0, \text { as } n \rightarrow \infty .
\end{aligned}
$$

Step 5: We can take a subsequence $u_{n_{i}}$ of $\left\{u_{n}\right\}$ and it is satisfied with

$$
\lim _{i \rightarrow \infty}\left\langle\delta g(t)-\bar{V} t, j\left(u_{n_{i}}-t\right)\right\rangle=\limsup _{n \rightarrow \infty}\left\langle\delta g(t)-\bar{V} t, j\left(u_{n}-t\right)\right\rangle .
$$

Since $X$ is a uniformly smooth Banach space and $\left\{v_{n}\right\}$ is bounded, there exists a subsequence $v_{n_{i}} \rightarrow \bar{v} \in C$ as $i \rightarrow \infty$. By (15) and (23), we know

$$
u_{n_{i}} \rightarrow \bar{v},\langle\delta g(t)-\bar{V} t, j(\bar{v}-t)\rangle=\limsup _{n \rightarrow \infty}\left\langle\delta g(t)-\bar{V} t, j\left(u_{n}-t\right)\right\rangle .
$$

From (21), (24) and Lemma 10, we can acquire $\bar{v} \in$ Fix (T). It follows that (22), (24) and Lemma 10, we can attain $\bar{v} \in$ Fix $(L)$. By (14), (24), Lemma 10 and Lemma 8, we get $\bar{v} \in V I(C, \widehat{B})$. So, we attain $\bar{v} \in \Psi$. From (24) and property of $Q_{C}$, we get

$$
\limsup _{n \rightarrow \infty}\left\langle\delta g(t)-\bar{V} t, j\left(u_{n}-t\right)\right\rangle=\langle\delta g(t)-\bar{V} t, j(\bar{v}-t)\rangle \leq 0 .
$$

Step 6: Finally, from Lemma 4 and Lemma 6, we acquire 


$$
\begin{aligned}
\left\|u_{n}-t\right\|^{2}= & \left\|\beta_{n} \delta g\left(v_{n}\right)+\left(I-\beta_{n} \bar{V}\right) L v_{n}-t\right\|^{2} \\
\leq & \left\|\left(I-\beta_{n} \bar{V}\right)\left(L v_{n}-t\right)\right\|^{2}+2 \beta_{n}\left\langle\delta g\left(v_{n}\right)-\bar{V} t, j\left(u_{n}-t\right)\right\rangle \\
\leq & \left(1-\beta_{n} \bar{\delta}\right)^{2}\left\|v_{n}-t\right\|^{2}+2 \beta_{n} \delta\left\langle g\left(v_{n}\right)-g(t), j\left(u_{n}-t\right)\right\rangle+2 \beta_{n}\left\langle\delta g(t)-\bar{V} t, j\left(u_{n}-t\right)\right\rangle \\
\leq & \left(1-\beta_{n} \bar{\delta}\right)^{2}\left\|v_{n}-t\right\|^{2}+2 \beta_{n} \delta\left\|g\left(v_{n}\right)-g(t)\right\|\left\|u_{n}-t\right\|+2 \beta_{n}\left\langle\delta g(t)-\bar{V} t, j\left(u_{n}-t\right)\right\rangle \\
\leq & \left(1-\beta_{n} \bar{\delta}\right)^{2}\left\|v_{n}-t\right\|^{2}+2 \beta_{n} \delta \eta\left\|v_{n}-t\right\|\left\|u_{n}-t\right\|+2 \beta_{n}\left\langle\delta g(t)-\bar{V} t, j\left(u_{n}-t\right)\right\rangle \\
\leq & \left(1-\beta_{n} \bar{\delta}\right)^{2}\left\|v_{n}-t\right\|^{2}+2 \beta_{n} \delta \eta\left\|v_{n}-t\right\|\left(\beta_{n}\left\|\delta g\left(v_{n}\right)-\bar{V} t\right\|+\left(1-\beta_{n} \bar{\delta}\right)\left\|L v_{n}-t\right\|\right) \\
& +2 \beta_{n}\left\langle\delta g(t)-\bar{V} t, j\left(u_{n}-t\right)\right\rangle \\
\leq & \left(1-\beta_{n} \bar{\delta}\right)^{2}\left\|v_{n}-t\right\|^{2}+2 \beta_{n} \delta \eta\left\|v_{n}-t\right\|\left(\beta_{n} \delta \eta\left\|v_{n}-t\right\|+\beta_{n}\|\delta g(t)-\bar{V} t\|\right. \\
& \left.+\left(1-\beta_{n} \bar{\delta}\right)\left\|v_{n}-t\right\|\right)+2 \beta_{n}\left\langle\delta g(t)-\bar{V} t, j\left(u_{n}-t\right)\right\rangle \\
\leq & \left(1-\beta_{n} \bar{\delta}\right)^{2}\left\|v_{n}-t\right\|^{2}+2 \beta_{n}^{2} \delta^{2} \eta^{2}\left\|v_{n}-t\right\|^{2}+2 \beta_{n}^{2} \delta \eta\|\delta g(t)-\bar{V} t\|\left\|v_{n}-t\right\| \\
& +2 \beta_{n} \delta \eta\left(1-\beta_{n} \bar{\delta}\right)\left\|v_{n}-t\right\|^{2}+2 \beta_{n}\left\langle\delta g(t)-\bar{V} t, j\left(u_{n}-t\right)\right\rangle \\
\leq & \left(1-\beta_{n} \bar{\delta}\right)\left\|v_{n}-t\right\|^{2}+2 \beta_{n}^{2} \bar{\delta}^{2}\left\|v_{n}-t\right\|^{2}+2 \beta_{n}^{2} \bar{\delta}\|\delta g(t)-\bar{V} t\|\left\|v_{n}-t\right\| \\
& +2 \beta_{n} \delta \eta\left\|v_{n}-t\right\|^{2}+2 \beta_{n}\left\langle\delta g(t)-\bar{V} t, j\left(u_{n}-t\right)\right\rangle,
\end{aligned}
$$

and

$$
\begin{aligned}
\left\|v_{n+1}-t\right\|^{2}= & \left\|\mu_{n} v_{n}+v_{n} T^{n}\left(\xi_{n} v_{n}+\left(1-\xi_{n}\right) v_{n+1}\right)+\omega_{n} Q_{C}(I-\lambda \widehat{B}) u_{n}-t\right\|^{2} \\
\leq & \mu_{n}\left\|v_{n}-t\right\|^{2}+v_{n} k_{n}^{2}\left\|\left(\xi_{n} v_{n}+\left(1-\xi_{n}\right) v_{n+1}\right)-t\right\|^{2}+\omega_{n}\left\|Q_{C}(I-\lambda \widehat{B}) u_{n}-t\right\|^{2} \\
\leq & \mu_{n}\left\|v_{n}-t\right\|^{2}+v_{n} k_{n}^{2} \xi_{n}\left\|v_{n}-t\right\|^{2}+v_{n} k_{n}^{2}\left(1-\xi_{n}\right)\left\|v_{n+1}-t\right\|^{2}+\omega_{n}\left\|u_{n}-t\right\|^{2} \\
\leq & \mu_{n}\left\|v_{n}-t\right\|^{2}+v_{n} k_{n}^{2} \xi_{n}\left\|v_{n}-t\right\|^{2}+v_{n} k_{n}^{2}\left(1-\xi_{n}\right)\left\|v_{n+1}-t\right\|^{2} \\
& +\omega_{n}\left[\left(1-\beta_{n} \bar{\delta}\right)\left\|v_{n}-t\right\|^{2}+2 \beta_{n}^{2} \bar{\delta}^{2}\left\|v_{n}-t\right\|^{2}+2 \beta_{n}^{2} \bar{\delta}\|\delta g(t)-\bar{V} t\|\left\|v_{n}-t\right\|\right. \\
& \left.+2 \beta_{n} \delta \eta\left\|v_{n}-t\right\|^{2}+2 \beta_{n}\left\langle\delta g(t)-\bar{V} t, j\left(u_{n}-t\right)\right\rangle\right] \\
\leq & \left(\mu_{n}+v_{n} k_{n}^{2} \xi_{n}+\omega_{n}-\omega_{n} \beta_{n} \bar{\delta}+2 \beta_{n} \delta \eta \omega_{n}\right)\left\|v_{n}-t\right\|^{2}+2 \beta_{n}^{2} \bar{\delta}^{2} \omega_{n}\left\|v_{n}-t\right\|^{2} \\
& +2 \beta_{n}^{2} \bar{\delta} \omega_{n}\|\delta g(t)-\bar{V} t\|\left\|v_{n}-t\right\|+2 \beta_{n} \omega_{n}\left\langle\delta g(t)-\bar{V} t, j\left(u_{n}-t\right)\right\rangle \\
& +v_{n} k_{n}^{2}\left(1-\xi_{n}\right)\left\|v_{n+1}-t\right\|^{2} .
\end{aligned}
$$

It follows that

$$
\begin{aligned}
\left(1-v_{n} k_{n}^{2}\left(1-\xi_{n}\right)\left\|v_{n+1}-t\right\|^{2} \leq\right. & \left(\mu_{n}+v_{n} k_{n}^{2} \xi_{n}+\omega_{n}-\omega_{n} \beta_{n}(\bar{\delta}-2 \delta \eta)\right)\left\|v_{n}-t\right\|^{2}+2 \beta_{n}^{2} \bar{\delta}^{2} \omega_{n}\left\|v_{n}-t\right\|^{2} \\
& +2 \beta_{n}^{2} \bar{\delta} \omega_{n}\|\delta g(t)-\bar{V} t\|\left\|v_{n}-t\right\|+2 \beta_{n} \omega_{n}\left\langle\delta g(t)-\bar{V} t, j\left(u_{n}-t\right)\right\rangle .
\end{aligned}
$$

That is 


$$
\begin{aligned}
\left\|v_{n+1}-t\right\|^{2} \leq & \frac{\mu_{n}+v_{n} k_{n}^{2} \xi_{n}+\omega_{n}-\omega_{n} \beta_{n}(\bar{\delta}-2 \delta \eta)}{1-v_{n} k_{n}^{2}\left(1-\xi_{n}\right)}\left\|v_{n}-t\right\|^{2}+\frac{2 \beta_{n}^{2} \bar{\delta}^{2} \omega_{n}}{1-v_{n} k_{n}^{2}\left(1-\xi_{n}\right)}\left\|v_{n}-t\right\|^{2} \\
& +\frac{2 \beta_{n}^{2} \bar{\delta} \omega_{n}}{1-v_{n} k_{n}^{2}\left(1-\xi_{n}\right)}\|\delta g(t)-\bar{V} t\|\left\|v_{n}-t\right\|+\frac{2 \beta_{n} \omega_{n}}{1-v_{n} k_{n}^{2}\left(1-\xi_{n}\right)}\left\langle\delta g(t)-\bar{V} t, j\left(u_{n}-t\right)\right\rangle \\
\leq & \left(1-\frac{\omega_{n} \beta_{n}(\bar{\delta}-2 \delta \eta)-v_{n}\left(k_{n}^{2}-1\right)}{1-v_{n} k_{n}^{2}\left(1-\xi_{n}\right)}\right)\left\|v_{n}-t\right\|^{2}+\frac{2 \beta_{n}^{2} \bar{\delta}^{2} \omega_{n}}{1-v_{n} k_{n}^{2}\left(1-\xi_{n}\right)}\left\|v_{n}-t\right\|^{2} \\
& +\frac{2 \beta_{n}^{2} \bar{\delta} \omega_{n}}{1-v_{n} k_{n}^{2}\left(1-\xi_{n}\right)}\|\delta g(t)-\bar{V} t\|\left\|v_{n}-t\right\|+\frac{2 \beta_{n} \omega_{n}}{1-v_{n} k_{n}^{2}\left(1-\xi_{n}\right)}\left\langle\delta g(t)-\bar{V} t, j\left(u_{n}-t\right)\right\rangle \\
\leq & \left(1-\frac{h_{1} \beta_{n}(\bar{\delta}-2 \delta \eta)-\varepsilon \beta_{n}}{1-v_{n} k_{n}^{2}\left(1-\xi_{n}\right)}\right)\left\|v_{n}-t\right\|^{2}+\frac{2 \beta_{n}^{2} \bar{\delta}^{2} \omega_{n}}{1-v_{n} k_{n}^{2}\left(1-\xi_{n}\right)}\left\|v_{n}-t\right\|^{2} \\
& +\frac{2 \beta_{n}^{2} \bar{\delta} \omega_{n}}{1-v_{n} k_{n}^{2}\left(1-\xi_{n}\right)}\|\delta g(t)-\bar{V} t\|\left\|v_{n}-t\right\|+\frac{2 \beta_{n} \omega_{n}}{1-v_{n} k_{n}^{2}\left(1-\xi_{n}\right)}\left\langle\delta g(t)-\bar{V} t, j\left(u_{n}-t\right)\right\rangle \\
\leq & \left(1-\frac{\beta_{n}\left(h_{1} \bar{\delta}-2 h_{1} \delta \eta-\varepsilon\right)}{1-v_{n} k_{n}^{2}\left(1-\xi_{n}\right)}\right)\left\|v_{n}-t\right\|^{2}+\frac{2 \beta_{n}^{2} \bar{\delta}^{2}}{1-v_{n} k_{n}^{2}\left(1-\xi_{n}\right)}\left\|v_{n}-t\right\|^{2} \\
& +\frac{2 \beta_{n}^{2} \bar{\delta}}{1-v_{n} k_{n}^{2}\left(1-\xi_{n}\right)}\|\delta g(t)-\bar{V} t\|\left\|v_{n}-t\right\|+\frac{2 \beta_{n}}{1-v_{n} k_{n}^{2}\left(1-\xi_{n}\right)}\left\langle\delta g(t)-\bar{V} t, j\left(u_{n}-t\right)\right\rangle \\
\leq & \left(1-\frac{\beta_{n}\left(h_{1} \bar{\delta}-2 h_{1} \delta \eta-\varepsilon\right)}{1-v_{n} k_{n}^{2}\left(1-\xi_{n}\right)}\right)\left\|v_{n}-t\right\|^{2}+\frac{\beta_{n}\left(h_{1} \bar{\delta}-2 h_{1} \delta \eta-\varepsilon\right)}{1-v_{n} k_{n}^{2}\left(1-\xi_{n}\right)}\left[\frac{2 \beta_{n} \bar{\delta}^{2}\left\|v_{n}-t\right\|_{1}^{2}}{h_{1} \bar{\delta}-2 h_{1} \delta \eta-\varepsilon}\right. \\
& \left.+\frac{2 \beta_{n} \bar{\delta}\|\delta g(t)-\bar{V} t\|\left\|v_{n}-t\right\|}{h_{1} \bar{\delta}-2 h_{1} \delta \eta-\varepsilon}+\frac{2\left\langle\delta g(t)-\bar{V} t, j\left(u_{n}-t\right)\right\rangle}{h_{1} \bar{\delta}-2 h_{1} \delta \eta-\varepsilon}\right] .
\end{aligned}
$$

Let

$$
\sigma_{n}=\frac{\beta_{n}\left(h_{1} \bar{\delta}-2 h_{1} \delta \eta-\varepsilon\right)}{1-v_{n} k_{n}^{2}\left(1-\xi_{n}\right)}
$$

and

$$
\theta_{n}=\frac{2 \beta_{n} \bar{\delta}^{2}\left\|v_{n}-t\right\|^{2}}{h_{1} \bar{\delta}-2 h_{1} \delta \eta-\varepsilon}+\frac{2 \beta_{n} \bar{\delta}\|\delta g(t)-\bar{V} t\|\left\|v_{n}-t\right\|}{h_{1} \bar{\delta}-2 h_{1} \delta \eta-\varepsilon}+\frac{2\left\langle\delta g(t)-\bar{V} t, j\left(u_{n}-t\right)\right\rangle}{h_{1} \bar{\delta}-2 h_{1} \delta \eta-\varepsilon},
$$

by conditions (i), (iii), (iv) in Theorem 1 and (25), we can gain

$$
\sum_{n=0}^{\infty} \sigma_{n}=\sum_{n=0}^{\infty} \frac{\beta_{n}\left(h_{1} \bar{\delta}-2 h_{1} \delta \eta-\varepsilon\right)}{1-v_{n} k_{n}^{2}\left(1-\xi_{n}\right)} \geq \sum_{n=0}^{\infty} \beta_{n}\left(h_{1} \bar{\delta}-2 h_{1} \delta \eta-\varepsilon\right)=+\infty,
$$

$\limsup _{n \rightarrow \infty} \theta_{n}=\limsup _{n \rightarrow \infty}\left[\frac{2 \beta_{n} \bar{\delta}^{2}\left\|v_{n}-t\right\|^{2}}{h_{1} \bar{\delta}-2 h_{1} \delta \eta-\varepsilon}+\frac{2 \beta_{n} \bar{\delta}\|\delta g(t)-\bar{V} t\|\left\|v_{n}-t\right\|}{h_{1} \bar{\delta}-2 h_{1} \delta \eta-\varepsilon}+\frac{2\left\langle\delta g(t)-\bar{V} t, j\left(u_{n}-t\right)\right\rangle}{h_{1} \bar{\delta}-2 h_{1} \delta \eta-\varepsilon}\right] \leq 0$.

Thus, from Lemma 5, we receive that $\lim _{n \rightarrow \infty}\left\|v_{n}-t\right\|=0$. So $\left\{v_{n}\right\}$ converges strongly to $t=$ $Q_{\Psi}(I-\bar{V}+\delta g) t \in \Psi$, and from Lemma 11, we have $(t, s, w)$ is a solution of the problem (4), where $s=Q_{C}\left(I-\lambda_{2} B_{2}\right)(b t+(1-b) w)$ and $w=Q_{C}\left(I-\lambda_{3} B_{3}\right) t$. The proof is completed.

As an application of our main result Theorem 1, we can prove strong convergence theorems for approximating the solution of the standard convex optimization problem.

Assume $C$ is a convex and closed subset of $X$. The standard constrained convex optimization problem is to find $\widetilde{v} \in C$ such that

$$
\widetilde{g}(\widetilde{v})=\min _{v \in C} \widetilde{g}(v),
$$

where $\widetilde{g}: C \rightarrow R$ is a convex, Frechet differentiable function. $\Phi_{\widetilde{g}}$ is used to indicate the solution set of (26). 
Lemma 12. ([21]) An essential condition of optimality for a point $\widetilde{v} \in C$ to be a solution of (26) is that $\widetilde{v}$ is a solution of the variational inequality

$$
\langle\nabla \widetilde{g}(\widetilde{v}), v-\widetilde{v}\rangle \geq 0, \forall v \in C
$$

Equivalently, $v \in C$ is a fixed point of the mapping $Q_{C}(I-\lambda \nabla \widetilde{g}), \forall \lambda>0$. In addition, if $\widetilde{g}$ is convex, then the optimality condition (27) is also sufficient.

Theorem 2. Assume that space $X$, mappings $Q_{C}, B_{1}, B_{2}, B_{3}, L, \bar{V}, g$ and $T$ are the same as in Theorem 1. Suppose $\widetilde{g}: C \rightarrow R$ is a convex real-valued function with the gradient $\nabla \widetilde{g}=\widehat{B}$ is $1 / L_{\widetilde{g}}$-inverse strongly accretive. Assume that $\Omega=\operatorname{Fix}(T) \cap \operatorname{Fix}(L) \cap \Phi_{\widetilde{g}} \neq \varnothing$. Define the sequence $\left\{v_{n}\right\}$ in $X$ as follows:

$$
\left\{\begin{array}{l}
v_{1} \in C \\
u_{n}=\beta_{n} \delta g\left(v_{n}\right)+\left(I-\beta_{n} \bar{V}\right) L v_{n}, \\
v_{n+1}=\mu_{n} v_{n}+v_{n} T^{n}\left(\widetilde{\xi}_{n} v_{n}+\left(1-\xi_{n}\right) v_{n+1}\right)+\omega_{n} Q_{C}(I-\lambda \nabla \widetilde{g}) u_{n}, n \geq 1
\end{array}\right.
$$

where $\lambda \in\left(0,2 / L_{\tilde{g}}\right), \beta_{n}, \mu_{n}, v_{n}, \omega_{n}, \xi_{n}$ and $\delta$ satisfy the same coefficient conditions as in Theorem 1. Then $\left\{v_{n}\right\}$ converges strongly to $t=Q_{\Omega}(I-\bar{V}+\delta g) t \in \Omega$ and $(t, s, w)$ is a solution of (4), where $s=$ $Q_{C}\left(I-\lambda_{2} B_{2}\right)(b t+(1-b) w)$ and $w=Q_{C}\left(I-\lambda_{3} B_{3}\right) t, \lambda_{1}, \lambda_{2}, \lambda_{3} \in(0,2 \bar{b})$ with $\bar{b}=\min \left\{b_{1}, b_{2}, b_{3}\right\}$.

Proof. From Lemma 12 and Theorem 1, we can gain Theorem 2, where $s=Q_{C}\left(I-\lambda_{2} B_{2}\right)(b t+(1-$ b) $w)$ and $w=Q_{C}\left(I-\lambda_{3} B_{3}\right) t$. Then, the proof is completed.

Remark 1. (1) In Theorem 2, we give the iterative approximation method and strong convergence results for the common elements of the solutions $\Phi_{\widetilde{g}}$ of the standard constrained convex optimization problem (26) and the fixed points Fix $(T)$ of an asymptotically non-expansive mapping $T$.

(2) As we all know, a Hilbert space $H$ is a uniformly smooth and uniformly convex Banach space and the metric projection $P_{C}: H \rightarrow C$ is sunny non-expansive retractive. So, when Hilbert space $H$ takes the place of Banach space $X$ and the sunny non-expansive retraction $Q_{C}$ becomes to the metric projection $P_{C}$, the results of Theorem 1 and Theorem 2 still hold. More specifically, if $k_{n} \equiv 1$ and $\xi_{n} \equiv 1$ in Theorem 2, we can attain the major results of Keerti et al. [19].

\section{Numerical Examples}

Someone may question whether the coefficients in Theorem 1 are complex enough to be not obtained. Now we give a concrete numerical example to show that the coefficients in Theorem 1 can be obtained.

Let $R$ be the real number set and $\widehat{B}, B_{1}, B_{2}, B_{3}$ be a mapping from $[0,20]$ to $R$ defined by $\widehat{B} v=$ $\frac{v}{4}, B_{1} v=\frac{v}{2}, B_{2} v=\frac{v}{4}, B_{3} v=\frac{v}{6}$, respectively. Put $b=\frac{1}{3}, \widehat{b}=4, b_{1}=2, b_{2}=4, b_{3}=6, \lambda_{1}=1, \lambda_{2}=$ $2, \lambda_{3}=3$, then $\bar{b}=\min \left\{b_{1}, b_{2}, b_{3}\right\}=2, \lambda_{1}, \lambda_{2}, \lambda_{3} \in(0,2 \bar{b})=(0,4)$, the mapping $L v=\frac{5}{18} v$ defined by (5) in Lemma 11. We take $\eta=\frac{1}{3}, \delta=\frac{1}{4}, \beta_{n}=\frac{1}{5 n}$, and let $g, \bar{V}: R \rightarrow R$ be defined by $g(v)=\frac{v}{3}, \bar{V} v=\frac{v}{4}$, respectively. So, $\left\{u_{n}\right\}: u_{n}=\frac{100 n+1}{360 n} v_{n}$ is generated by (6). Assume that $T$ is an asymptotically non-expansive mapping defined by $T^{n_{v}}=\sqrt{1+\frac{1}{12 n}} v$. Let $v_{1} \in[0,20]$, and $\left\{v_{n}\right\}$ is generated by (6) where $h_{1}=\frac{1}{6}, h_{2}=\frac{1}{2}, \lambda=1 \in(0,2 \widehat{b})=(0,8), \mu_{n}=\frac{3 n-1}{12 n}, v_{n}=\frac{1}{2}$ and $\omega_{n}=$ $\frac{3 n+1}{12 n}, \xi_{n}=1-\frac{1}{3 n}, \varepsilon=\frac{5}{12}, \tau=\frac{3}{4}$. From the propose definition, the all conditions of Theorem 1 are satisfied. We know $0 \in \operatorname{Fix}(T) \cap \operatorname{Fix}(L) \cap V I(C, \widehat{B})$. Then $v_{n}$ is generated by (6) and we simplify the form of (6) to get

$$
v_{n+1}=\frac{1440 n^{2}-480 n+480 n \sqrt{3 n(12 n+1)}-160 \sqrt{3 n(12 n+1)}+1}{5760 n^{2}-160 \sqrt{3 n(12 n+1)}} v_{n} \approx \frac{4320}{5760} v_{n} .
$$


So, clearly, $v_{n}$ converges strongly to $0 \in \operatorname{Fix}(T) \cap \operatorname{Fix}(L) \cap V I(C, \widehat{B})$.

\section{Conclusions}

In this paper, we present a new generalized variational inequality system problem (4) which contains many special cases. In the case when we put $B_{2} \equiv 0$, then the problem (4) reduces to problem (3); If putting $b=0$ in (4), we get

$$
\left\{\begin{array}{l}
\left\langle t-\left(I-\lambda_{1} B_{1}\right) s, j(v-t)\right\rangle \geq 0, \forall v \in C, \\
\left\langle s-\left(I-\lambda_{2} B_{2}\right) w, j(v-s)\right\rangle \geq 0, \forall v \in C, \\
\left\langle w-\left(I-\lambda_{3} B_{3}\right) t, j(v-w)\right\rangle \geq 0, \forall v \in C,
\end{array}\right.
$$

which is a variational inequality modified system by Ceng et al. [2]. In the sense that if we take $B_{3} \equiv 0$ in (29), then the problem (29) reduces to problem (2). We study the common elements of the set of solutions of an asymptotically non-expansive operation equation with the $L$ mapping defined by (5) and the solution set of the generalized proposed system problem (4). The convergence analysis of a new way by using the generalized semi-closure principle supplied by us in Wang et al. [9] for finding the propose common elements in Banach spaces are investigated. Under the suitable conditions imposed on parameters, some strong convergence theorems are attained. We locally generalize some corresponding recent results of Keerti et al. [19] from Hilbert spaces to Banach spaces and from non-expansive mapping to asymptotically non-expansive mapping. As an application, we prove the strong convergence theorem of the standard constrained convex optimization problem by our main result. Eventually, we also give a real numerical example to show that the coefficients in Theorem 1 can be obtained and the theorems are reasonable and valid.

Author Contributions: conceptualization, C.L.; data curation, C.L. and L.L.; formal analysis, Y.W. and C.L.; funding acquisition, Y.W. and C.L.; investigation, Y.W. and L.L.; methodology, Y.W., C.L. and L.L.; project administration, Y.W.; resources, C.L.; Software, L.L.; supervision, Y.W.; validation, L.L.; writing-original draft, C.L. and L.L. All authors have read and agreed to the published version of the manuscript.

Funding: This research received no external funding.

Acknowledgments: This work was supported by the National Natural Science Foundation of China (Grant no. 11671365) and the Natural Science Foundation of Zhejiang Province (Grant no. LY14A010011).

Conflicts of Interest: The authors declare that they have no competing interests.

\section{References}

1. Blum, E.; Oettli, W. From optimization and variational inequalities to equilibrium problems. Math. Stud. 1994, 63, 123-145.

2. Ceng, L.C.; Wang, C.Y.; Yao, J.C. Strong convergence theorems by a relaxed extragradient method for a general system of variational inequalities. Math. Methods Oper. Res. 2008, 67, 375-390. [CrossRef]

3. Imnang, S. Viscosity iterative method for a new general system of variational inequalities in Banach Spaces. J. Inequal. Appl. 2013, 2013, 249. [CrossRef]

4. Zhang, H.C.; Qu, Y.H.; Su, Y.F. Strong convergence theorems for fixed point problems for nonexpansive mappings and zero point problems for accretive operators using viscosity implicit midpoint rules in Banach Spaces. Mathematics 2018, 6, 257. [CrossRef]

5. Noor, M.A. On iterative methods for solving a system of mixed variational inequalities. Appl. Anal. 2008, 87, 99-108. [CrossRef]

6. Qin, X.; Cho, S.Y.; Kang, S.M. Convergence of an iterative algorithm for systems of variational inequalities and nonexpansive mappings with applications. J. Comput. Appl. Math. 2009, 233, 231-240. [CrossRef]

7. Yao, Y.; Liou, Y.C.; Kang, S.M. Approach to common elements of variational inequality problems and fixed point problems via a relaxed extragradient method. Comput. Math. Appl. 2010, 59, 3472-3480. [CrossRef]

8. Yao, Y.; Maruster, S. Strong convergence of an iterative algorithm for variational inequalities in Banach spaces. Math. Comput. Model. 2011, 54, 325-329. [CrossRef] 
9. Pan, C.J.; Wang, Y.H. Generalized viscosity implicit iterative process for asymptotically non-expansive mappings in Banach spaces . Mathematics 2019, 7, 379. [CrossRef]

10. Xu, H.K. Viscosity approximation methods for nonexpansive mappings. J. Math. Anal. Appl. 2004, 298, 279-291. [CrossRef]

11. Kim, K.S. Convergence to common solutions of various problems for nonexpansive mappings in Hilbert spaces. Fixed Point Theory Appl. 2012, 2012, 185. [CrossRef]

12. Liu, Y. Weak convergence of a new projection algorithm for variational inequalities in Banach spaces. Nonlinear Funct. Anal. Appl. 2016, 21, 121-129.

13. Matveev, S.A.; Stadnichuk, V.I.; Tyrtyshnikov, E.E.; Smirnov, A.P.; Ampilogova, N.V.; Brilliantov, N.V. Anderson acceleration method of finding steady-state particle size distribution for a wide class of aggregation-fragmentation models. Comput. Phys. Commun. 2018, 224, 154-163. [CrossRef]

14. Timokhin, I.V.; Matveev, S.A.; Siddharth, N.; Tyrtyshnikov, E.E.; Smirnov, A.P.; Brilliantov, N.V. Newton method for stationary and quasi-stationary problems for Smoluchowski-type equations. J. Comput. Phys. 2019, 382, 124-137. [CrossRef]

15. Brezinski, C. Redivo-Zaglia, M. The Simplified Topological epsion-Algorithms for Accelerating Sequences in a Vector Space. SIAM J. Sci. Comput. 2014, 36, A2227-A2247. [CrossRef]

16. Walker, H.F.; Ni, P. Anderson acceleration for fixed point iterations. SIAM J. Numer. Anal. 2011, 49, 1715-1735. [CrossRef]

17. Katchang, P.; Kumam, P. Convergence of iterative algorithm for finding common solution of fixed points and general system of variational inequalities for two accretive operators. Thai J. Math. 2011, 9, 343-360.

18. Wang, Y.H.; Pan, C.J. Viscosity approximation methods for a general variational inequality system and fixed point problems in Banach spaces. Symmetry 2020, 12, 36. [CrossRef]

19. Keerati, S.; Atid, K. A new general system of variational inequalities for convergence theorem and application. J. Appl. Math. 2019, 81, 99-123.

20. Noor, M.A. Some algorithms for general monotone mixed variational inequalities. Math. Comput. Model. 1999, 29, 1-9. [CrossRef]

21. Su, M.; Xu, H.K. Remarks on the Gradient-Projection algorithm. J. Nonlinear Anal. Optim 2010, 1, 35-43.

22. Yao, Y.; Postolache, M.; Yao, J.C. An iterative algorithm for solving the generalized variational inequalities and fixed points problems. Mathematics 2019, 7, 61. [CrossRef]

23. Zegeye, H.; Ofoedu, E.; Shahzad, N. Convergence theorems for equilibrium problem, variational inequality problem and countably infinite relatively quasi-nonexpansive mappings. Appl. Math. Comput. 2010, 216, 3439-3449. [CrossRef]

24. He, Z.; Du, W.-S. Strong convergence theorems for equilibrium problems and fixed point problems: A new iterative method, some comments and applications. Fixed Point Theory Appl. 2011, 2011, 15. [CrossRef]

25. He, Z.; Du, W.-S. Viscosity Iterative Schemes for Finding Split Common Solutions of Variational Inequalities and Fixed Point Problems. Abstr. Appl. Anal. 2012, 2012, 25. [CrossRef]

26. He, Z.; Du, W.-S. On hybrid split problem and its nonlinear algorithms. Fixed Point Theory Appl. 2013, 2013, 47. [CrossRef]

27. Cai, G.; Hu, C.S. Strong convergence theorems of a general iterative process for a finite family of $\lambda_{i}$-strict pseudo-contractions in q-uniformly smooth Banach spaces. Comput. Math. Appl. 2010, 59, 149-160. [CrossRef]

28. Aoyama, K.; Iiduka, H.; Takahashi, W. Weak convergence of an iterative sequence for accretive operators in Banach spaces. Fixed Point Theory Appl. 2006, 2006, 35390. [CrossRef]

Publisher's Note: MDPI stays neutral with regard to jurisdictional claims in published maps and institutional affiliations.

(C) 2020 by the authors. Licensee MDPI, Basel, Switzerland. This article is an open access article distributed under the terms and conditions of the Creative Commons Attribution (CC BY) license (http:/ / creativecommons.org/licenses/by/4.0/). 